\title{
BIBLIOGRAFIA
}

\section{Bibliografía de Georges Alfred Cirot}

Fsta bibliografía de los trabajos, artículos y estudios de un distinguido hispanista francés, el finado $M$. Georges Alfred Cirot, fué compilada principalmente del Bulletin Hispanique, publicado por la Faculté des Lettres de la Universidad de Bordeaux, Francia. Este periódico, publicado en la ciudad de Bordeaux desde 1898, está consagrado a los estudios hispánicos de todo el mundo. No obstante su larga vida y su importancia - y esto es difícil explicarlo- la circulación del Bulletin Hispanic en Norteamérica es bastante reducida. ${ }^{1}$ A la vez, según parece, los trabajos de $\mathrm{M}$. Cirot han permanecido también, de hecho, ignorados. A mi entender, el influjo de M. Cirot en las letras hispanofrancesas es de bastante importancia, y debe darse la mayor publicidad a su trabajo, como scholar, maestro y editor. Por esa razón compilé esta bibliografía.

Ningún escritor puede hacer algún trabajo de investigación, sin haber formado antes la correspondiente lista bibliográfica. Como esa lista no es un fin sino una fácil y eficaz manera de preparar una tarea más larga, importa darle el valor que tiene, como simple instrumento de trabajo.

El mejor método de clasificación para la extensa bibliografía de M. Cirot, me ha parecido que era el orden cronológico. De acuerdo con él, ha sido ordenada por años. Así, el lector estará en condiciones de apreciar, de una ojeada, la actividad que desplegó el maestro de estudios hispánicos en más de media centuria, desde la época en que fué nombrado Maître de Conférences de la Universidad de Bordeaux, en 1896, hasta su muerte acaecida en 1946. Dentro de cada 
año, los trabajos de M. Cirot -libros, estudios, artículos, ediciones de textos y prólogos- están clasificados por orden alfabético de títulos; las reseñas críticas van separadas de los demás trabajos y clasificadas, alfabéticamente, por autơres de los trabajos reseñados. Cuando se sabe que un estudio de M. Cirot apareció en otra parte, además del Bulletin Hispanique, el nombre del periódico o del trabajo se da, junto con el año, volumen y página donde puede hallarse. Va al final un índice general alfabético de personas y asuntos, para facilitar la consulta de cualquier autor o trabajo importante.

No he incluído en esta bibliografía trabajo crítico alguno, acerca de M. Cirot, a excepción del artículo publicado en el Bulletin Hispanique $^{2}$ por M. Charles V. Aubrun, y el destinado a Hispania ${ }^{3}$ por el suscrito. Estoy seguro de que, al fallecer M. Cirot, dedicaron abundantes artículos, tanto en Francia como en España, a su vida y a su obra. Como no he visto muchos de ellos, ni podría enumerarlos aquí, debo contentarme con publicar la bibliografía de sus trabajos; tributo que, después de todo, resulta de igual o mayor importancia que las notas encomiásticas escritas a su muerte.

Doy las más sinceras gracias a la señorita Helen Conover, de la Biblioteca del Congreso, por sus valiosas sugestiones, y al profesor Homero Serís, del Centro de Estudios Históricos de la Universidad de Syracuse, por haber leído el original de mi trabajo y haberle hecho importantes críticas. Como esta bibliografía se halla muy lejos de considerarse completa, el compilador agradecerá cualquier indicación que se le haga, sobre lagunas e imperfecciones de su trabajo.

Henry V. Besso, M. A.

\section{NOTAS}

1. Union List of Serials in the United States and Canada, por Winifred Gregory. La segunda edición (New York, H. W. Wilson) y el Suplemento, ambos publicados en 1943, indican que sólo cincuenta y seis (56) universidades y bibliotecas, a lo largo de los Estados Unidos y el Canadá, se han suscrito a este periódico, y que las colecciones, en más del cincuenta por ciento 
de los casos, se hallan incompletas. Este descubrimiento me ha sorprendido y decepcionado.

2. Bulletin Hispanique, 1946, XLVIII, no, 4, 289-293.

3. Hispania, Washington, D. C. November, 1947. 



\title{
Bibliografía de los escritos de Georges Cirot
}

\author{
$\begin{array}{llll}1 & 8 & 9 & 4\end{array}$
}

1. Synchronismes de la littérature française depuis les origines jusqu'à nos jours, en 44 tableaux, suivis d'une table alpbabétique, à l'usage des candidats aux divers examens de lettres (brevet supérieur, baccalauréat et licence), par G. Cirot, A. Dufourcq et R. Thiry. Paris, Bloud et Barral, (1894), 64 pp.

\section{8}

2. Episcologio ampuritano (D. Ramon Fort); La genèse des exercises spirituels de Saint Ignace de Loyola (H. Watrigant). En: Revue des Universités du Midi, IV, 32.

3. Religióes da Lusitania (J. Leite de Vasconcellos). En: Revue des Universités du Midi, Iv, 229.

\section{9}

4. Les études bispaniques à l'Université de Bordeaux. En: Bulletin Hispanique, * $\mathrm{I}, 255$.

5. Un nouveau roi Wisigoth. $\mathrm{BH}, \mathrm{I}, 41-45$.

R e s e. $\tilde{n}$ a $s:$

6. Bello, Andrés. Gramática de la lengua castellana destinada al uso de los americanos: sexta edición hecha sobre la última del autor con extensas notas y un copioso indice alfabético de D. Rufino José Cuervo. BH. I, núm. 4, 230-233.

* A continuación, abreviado $B H$. 
7. Castro, Adolfo de. Libro de los galicismos. Madrid, La España Moderna. BH. I, 85-87.

1900

8. Irissary Honorat, B. Estudio completo de la conjugación de la lengua francesa. BH, II, 324-326.

9. Macé, Alcide. De emendando Differentiarum libro qui inscribitur "De proprietate sermonum." BH, II, núm. 4, 311-313. (With E. Bourciez.)

10. Menéndez Pidal, Ramón. Catálogo de la real Biblioteca. BH, I, 110-114.

11. Verdaguer, Mosén Jacinto. Canigó, versión castellana por el Conde de Cedillo. $\mathrm{BH}, \mathrm{I}, 213-214$.

\section{$\begin{array}{llll}1 & 9 & 0 & 1\end{array}$}

12. Les éditions de l'Historia de España de Mariana. $\mathrm{BH}$, IIr, núm. 1, 83-85.

13. La Festa d'Elche. BH, III, 313.

14. Nuevos datos acerca del histrionismo español en los siglos XVI y $X V I I$, recogidos por $D$. Cristóbal Pérez Pastor. Madrid, Imprenta de la Revista Española, 1901, 418 pp. [A second. serie, published with and Index by Georges Cirot. Bordeaux, Féret \& Fils, 1914. xxII, 219 pp. ('Elle n'est qu'un tiré à part (à 50 exemplaires) d'une publication commencée par le Bulletin Hispanique en 1906 et terminée seulement en 1914."' Pref.)]

Res e $\tilde{n}$ a $s:$

15. Brito Aranha, etc. Le Portugal. Paris, Larousse, s. d. 368 pp. BH, III, 78-79.

16. Férotin, Dom Marius. Apringius de Beja, son commentaire sur l'Apocalypse. Paris, Alphonse Picard, 1900. xxrv-90 pp. BH, m, 66-67.

17. L'Espagne. Politique, littérature; armée et marine, justice, enseignement, économie, ethnograpbie, colonies, beaux-arts; la cour, la 
société, etc. Número special encyclopédique de la Nouvelle Revue Internationale. $\mathrm{BH}, \mathrm{mI}, 79$.

\section{$\begin{array}{llll}19 & 0 & 2\end{array}$}

18. Sur un procédé de style de Francisco de Melo. BH, IV, núm. 2, 163-166.

\section{3}

R e $s$ e ñ a s :

19. Unamuno, Miguel de. En torno al casticismo. Madrid, Fernando Fe, 1902. 212 pp. (Biblioteca de Ciencias Sociales.) BH, v, 198.

$$
1904
$$

20. Études sur l'bistoriographie espagnole. Les bistoires générales entre Alphonse X et Philippe II (1284-1556). Bordeaux, Féret \& Fils, 1904. 180 pp. (Thèse secondaire. Université de Paris.) ${ }^{1}$

21. La famille de Juan de Mariana. BH, vI, 309-331.

22. "Ser" et "estar" avec un participe passé. En: Mélanges de pbilologie offerts à Ferdinand Brunot. Paris, 1904, pp. 57-69.

$$
1905
$$

23. Etudes sur l'bistoriograpbie espagnole. Mariana, bistorien. Bordeaux, Féret \& Fils, 1905. xiv, 481 pp. (Bibliothèque de la Fondation Thiers VIII.) (Issued also as a Thesis: Paris, 1904). ${ }^{2}$

24. Les portraits du P. Juan de Mariana. BH, vn, 409-411.

$R$ e s e $\tilde{n}$ a $s$ :

25. Catálogo de la exposición celebrada en la Biblioteca Nacional en el tercer centenario de la publicación del Quijote. Año 1905. Madrid, Imprenta alemana, s. a. núm. 1. BH, vir, 216.

1906

26. Barrau-Dibigo, L. BH, viI, núm. 3, 326-327. (Contestación de M. Cirot a una carta de M. Barrau-Dihigo.) 
27. Des noms et des prénoms. BH, vir, 190-191. (Respuesta a una crítica de M. Barrau-Dihigo sobre un estudio de Cirot, publicada en la Revue Hispanique, 1905.)

28. Documents sur le faussaire Higuera. $\mathrm{BH}$, vm, 87-95.

29. Recherches sur les Juifs espagnols et portugais à Bordeanx. I. Les vestiges de l'espagnol et du portugais dans le parler actuel des juifs bordelais. BH, vur, 172-189; 279-296; 383-391. (Seguirá publicándose.) ${ }^{3}$

\section{7}

30. Quelques remarques sur les archaïsmes de Mariana et la langue des prosateurs de son temps (conjugaison). En: Romanische Forschungen. Erlangen, Band 23, 885-904.

31. Recherches sur les Juifs espagnols et portugais à Bordeaux... BH, Ix, 41-66;263-276;386-400.

32. Notes bibliograpbiques sur les auteurs et les questions du programme pour le concours de 1907. BH, Ix, 92-102; 416-428. (Con E. Martinenche y E. Mérimée.)

R e $s$ e $\tilde{\mathbf{n}}$ a s :

33. Pulido y Fernández, Angel. Intereses nacionales. Españoles sin patria y la raza sefardi. Madrid, E. Teodoro, 1905 . viii-659 pp. BH, IX, $429-436$.

34. Ribera, Julián. Lo cientifico en la bistoria. Madrid, Apalategui, 1906. 191 pp. BH, Ix, 437.

35. Schiff, Mario. La Bibliothèque du Marquis de Santillane. Paris, Bouillon, 1905. xci-509 pp. BH, Ix, 312-314.

36. Vergara y Martín, G. M. Ensayo de una colección bibliográficabiográfica de noticias referentes a la provincia de Segovia. Guadalajara, 1904. 616 pp. BH, Ix, 436-437.

$$
1908
$$

37. A propos du "De rege", des "Septem Tractains" de Mariana et de son, ou de ses procès. $\mathrm{BH}, \mathrm{x}, 95-99$. 
38. Notes bibliographiques sur les auteurs et les questions du programme pour le concours de 1909. (Con E. Mérimée y E. Martinenche. $\mathrm{BH}, \mathrm{x}, 417-428$.

39. Recherches sur les Juifs espagnols et portugais à Bordeaux ... BH, $\mathrm{x}, 68-86 ; 157-192 ; 259-285 ; 353-367$.

\section{9}

40. Une chronique léonaise inédite. $\mathrm{BH}, \mathrm{xI}, 259-282$.

41. Les décades d'Alfonso de Palencia, la Cbronique castillane de Henri IV attribuée à Palencia et le "Memorial de diversas bazañas" de Diego de Valera. BH, XI, 425-442.

42. Les Juifs de Bordeaux. Leur situation morale et sociale de 1550 a la Revolution. En: Revue Historique de Bordeaux, n, 368-382; IV, 145-166; vII, 353-370; viIr, 22-38, 169-185, 267-275; IX, 23-36, 203-220; XI, 129-142, 200-207; XII, 14-28. ${ }^{4}$

43. Notes bibliographiques sur les questions du programme pour le concours de 1910. (Con E. Mérimée, E. Martinenche y A. MorelFatio.) $\mathrm{BH}, \mathrm{xI}, 448-450$.

R e $s$ e $\tilde{n}$ a $s$ :

44. Pedrell, F. Catálech de la Biblioteca musical de la Diputació de Barcelona ab notes bistóriques, biográfiques et critiques, transcripcions en notació moderna dels principals motius musicals $y$ fascimils dels documents més importants pera la bibliografia espanyola, per... V. x. Barcelona, Palau de la Diputació, 1907, 330 pp. BH, XI, 338.

45. Salarrullana de Dios, José. Colección de documentos para el estudio de la bistoria de Aragón. Tomo III. Documentos correspondientes al reinado de Sancio Ramires (Vol. I) (1063-1094.) Zaragoza, Tipografía de Escar, 1907. 267 pp. BH, xI, 118-119.

\section{0}

46. Notes bibliograpbiques sur les questions du programme pour le concours de 1911. (Con E. Mérimée.) BH, xrr, 437-438. 
47. L'Université de Bordeaux et le Portugal. BH, xII, 339-341.

48. La visite des universitaires espagnols à Bordeaux. BH, XII, 344-346.

$R$ e $s$ e $\tilde{n}$ a $s$ :

49. Coulet, J. Étude sur l'office de Girone en l'bonneur de Saint Charlemagne. Montpellier, Coulet, 1907. 165 pp. BH, XII, 101-105.

50. Leite de Vasconcellos, J. Textos archaicos. $2^{a}$ edição (ampliada). Lisboa, Texeira, 1908. 160 pp. BH, xrr, 444.

S1. Mérimée, Henri. El prado de Valencia, édition critique avec une introduction, des notes et un appendice. Toulouse, Privat, 1907. 239 pp. BH, xII, 239-242.

52. Picón-Febres, G. La literatura venezolana en el siglo XIX. Caracas, 1906. BH, XII, 244.

1911

53. La Cbronique léonaise (suite). $\mathrm{BH}, \mathrm{xm}, 381-439$.

54. Diplôme d'études supérieures. BH, xIIr, 235.

55. Notes bibliographiques sur les questions du programme pour le concours de 1912. (Con E. Mérimée y E. Martinenche.) BH, xm, 468474.

Res e $\tilde{\mathbf{n}}$ a $s$ :

56. Eyzaguirre Rouse, G. Crónica general de España por Fray García de Euguy, transcripción becha del MS. del Escorial por... ( $\mathbf{P u}_{\mathrm{u}}$ blicada en los Anales de la Universidad de Chile). n. d. BH, xm, 237-238.

57. Labra, R. M. de. La orientación internacional de España. Madrid, Alfredo Alonso, 1910. 320 pp. BH, xum, 378-379.

58. Maura, G. Rincones de la bistoria. Apuntes para la bistoria social de España. Tomo I. Siglo VIII al XIII. Madrid, Fr. Beltrán, 1910. 378 pp. BH, xIII, 373-374.

59. Toro Gisbert, M. de. Apuntaciones lexicográficas. Paris, Ollendorf, s. d. 289 pp. BH, XIII, 502 . 
60. Ugarte, Manuel. El porvenir de la América latina. Valencia, Sampere, s. d. (1911). 321 pp. BH, xur, 379.

\section{2}

61. Une chronique latine inédite des Rois de Castille (1236). $\mathrm{BH}$, XIv, $30-46 ; 109-118 ; 244-274 ; 353-374$.

62. Commentaire d'une page de la Ciencia española. Madrid, 1912.7 pp. (De la Revista de Archivos, Bibliotecas y Museos.)

63. Diplômes d'études supérieures d'espagnol (Con E. M.) BH, xIv, 102.

64. L'espagnol dans l'enseignement secondaire. $\mathrm{BH}, \mathrm{xIV}, 444-445$.

65. Herculano et l'bistoire des Arabes. BH, xIv, 208.

66. L'intercambio avec le Portugal. BH, xiv, 101-102.

R e s e $\tilde{\mathbf{n}}$ a $s$ :

67. Antolín, G. Catálogo de los Códices latinos de la R. Biblioteca del Escorial. V. I. (liii-576 pp.), 1910; Vol. II (596 pp), 1911. Madrid, Imprenta helénica. BH, xIv, 448-449.

68. Colleção de manuscriptos ineditos agora dados a estampa. I. O livro la Corte Imperial. II. O livro da virtuosa bemfeitoria do Infante Dom Pedro. III. Fastigimia por Thomé Pinheiro da Veiga. Porto, 1910-1911. 308, 274, 374 pp. BH, XIV, 103-105.

69. Fueter, E. Geschichte der neueren Historiograpbie. München und Berlín, R. Oldenbourg, 1911. xx-626 pp. BH, xIv, $449-454$.

70. Morley, Griswold. Spanish ballads (romances escogidos) edited with introduction, notes and vocabulary by ... New York, Henry Holt, 1911. 226 pp. BH, xrv, 225.

\section{3}

71. De operibus bistoricis Jobonnis Aegidii Zamorensis qui tempore Aldephonsi Decimi regis Castellae scribebet, etc. Burdigalae, apud $\mathrm{Fe}-$ ret, 1913.81 pp. (Bibliotheca Latina medii aevi, I.)

72. Inauguration de l'Institut français de Madrid. BH, xv, 340-341. 
73. Notes bibliographiques sur les questions et auteurs des programmes d'agrégation et de certificat secondaire pour la langue espagnole en 1913. (Con E. Mérimée y E. Martinenche.) BH, xv, 93-99.

74. Notes bibliographiques sur les questions et auteurs des programmes d'agrégation et de certificat secondaire pour la langue espagnole en 1914. (Con H. M. y H. G.) BH, xv, 470-476.

75. Les professeurs d'Oviedo à Bordeaux. BH, xv, 348-349.

R e s e ñ a s :

76. Bernard, Augustin. Le Maroc. Paris, Alcan, 1903.412 pp. BH, xv, 111.

77. Bona, R. Essai sur le problème mercantiliste en Espagne au XVIIe siècle. Bordeaux, Condorcet, 1911. 209 pp. (Thèse pour le doctorat, soutenue devant la Faculté de Droit de Bordeaux.) $\mathrm{BH}, \mathrm{xv}, 488$ 489.

78. Bratli, Ch. Philippe II, roi d'Espagne. Étude sur sa vie et son caractère. Paris, Champion, 1912. 309 pp. BH, xv, 487-488.

79. Cejador y Frauca, Julio. Arcipreste de Hita, Libro de Buen Amor. Edición y notas de ... Madrid, Ediciones de "La Lectura", 1913. 2 vols. $\mathrm{BH}, \mathrm{xv}, 479-484$.

80. Morel-Fatio, A. Historiograpbie de Charles Quint. Première partie suivie des Mémoires de Charles-Quint, texte portugais et traduction française. Paris, Champion, 1913. 369 pp. (CCIIe fascicule de la Bibliothèque de l'École des Hautes Études.) $\mathrm{BH}, \mathrm{xv}, 350-358$.

\section{4}

81. Biograpbie du Cid, par Gil de Zamora (XIIle siècle). $\mathrm{BH}$, xvr, 80-86.

82. La Chronique léonaise et la Chronique dite de Silos. $\mathrm{BH}, \mathrm{xvI}, 15-34$.

83. L'espagnol au baccalauréat. $\mathrm{BH}, \mathrm{xvi}, 88-91$.

84. Florian de Ocampo, chroniste de Charles-Quint. BH, XvI, 307-336.

85. Lorenzo de Padilla et la pseudo-bistoire. BH, xvI, 405-447. 
86. Sur un tableau au dos duquel il y a une inscription espagnole l'attribuant à Rapbael. $\mathrm{BH}, \mathrm{xvI}, 391-397$.

R e s e ñ a s :

87. Babelon, J. La Bibliothèque française de Fernand Colomb. Paris, Champion, 1913. 341 pp. BH, xvI, 491-492.

88. Burnam. J. M. Palaeograpbia ibérica. Fac-similés de manuscrits espagnols et portugais (IX-XVe siècles). 1er fascicule. Paris, Champion, 1912. 80 pp. BH, xvi, 95-98.

89. Carreras y Candi, F. Geografia general de Catalunya dirigida por... Catalunya. Descripció politica-bistórica-social por . . BH, xvi, 493.

90. Daumet, G. Mémoire sur les relations de la France et de la Castille de 1255 à 1320. Paris, Fontemoing, 1913. 257 pp. BH, xvI, 489490.

91. Holle, Fritz ed. Mateo Alemán, Guzmán de Alfarache. $1^{\text {a }}$ parte. BH, xVI, $116-117$.

92. Ibarra Rodríguez, E. Colección de documentos ,para el estudio de la bistoria de Aragón, tomo IX. Documentos correspondientes al reinado de Sancio Ramires (1063-1093). BH, xvI, 488-489.

93. López, P. Savj. Cervantes. Napoli, Riccardo Ricciardi, 1913. 247 pp. BH, XvI, 117.

94. Magnin, E. L'Église wisigothique au VIle siècle. Tomo I. Paris, A. Picard, 1912. 204 pp. (Bibliothéque d'Histoire réligieuse.) $\mathrm{BH}, \mathrm{xvI}$, 93-95.

95. Mir, Miguel. Santa Teresa de Jesús, su vida, su espiritu, sus fundaciones. Madrid, Rates. 2 tomos. xxviii-805 y 847 pp. BH, xvI, 98116.

96. Mir, Miguel. Historia interna documentada de la Cía de Jesús. Madrid, Rates, 1913. 2 tomos, 68-520 y 852 pp. BH, xvI, 98-116.

97. Orueta, R. de. La vida y la obra de Pedro de Mena y Medrano. Madrid, Imprenta de Blass y Cía., 1914. 340 pp. BH, xvI, 492-493. 
98. Prestage, E. et Pedro d'Azevedo. Registos parochiaes de Lisboa. Registo da Freguesia de Santa Cruz do Castello desde 1536 até 1628 publicado com introducção, notas e indice por ... Coimbra, Imprensa da Universidade, 1913. 343 pp. BH, xvr, 255-256.

99. Robles Dégano, F. Los disparates gramaticales de la real Academia española y su corrección. Madrid, Fernando Fe, 1912. 88 pp. BH, $\mathrm{xvI}, 130$.

100. Rosenberg, S. L. Millard. Las burlas veras, comedia de Lope de Vega Carpio, edited with an introduction and notes by... Philadelphia, 1912. 94 pp. (Publications of the University of Pennsylvania. Dept. of Romanic Languages and Literatures.) BH, xvт, 122.

101. Salvá, V. Nuevo diccionario francés-español y español-francés. $\mathrm{Pa-}$ ris, Garnier, s. d. 705 and 838 pp. BH, xvt, 128-129.

102. Sorrento, L. ed. La vida de Lazarillo de Tormes. Fasc. 177 de la Bibliotbeca románica. $\mathrm{BH}, \mathrm{xvI}, 116-117$.

103. Toro y Gisbert, M. S. Pequeño Larousse ilustrado. Nuevo diccionario enciclopédico, publicado bajo la dirección de Claude Augé, adaptación española de Miguel de Toro y Gisbert. Paris, Larousse, 1913. $1528 \mathrm{pp} . \mathrm{BH}, \mathrm{xvI}, 129-130$.

\section{5}

104. Figueiredo, F. de. O espirito historico. Introducção a Bibliotheca. Noçoes preliminares. $2^{a}$ edição seguida duma Bibliographia portugueza de Theoria e Ensino da Historia. Lisboa, Texeira, 1915. BH, XVIr, 156.

\section{6}

105. La Cbronique léonaise et les Chroniques de Sebastien et de Silos. $\mathrm{BH}, \mathrm{xvII}, 1-25$.

106. La Chronique léonaise et les Chroniques de Pelage et de Silos. BH, XVIII, 141-154.

107. Diplômes d'études supérieures. $\mathrm{BH}, \mathrm{xvm}$, 54-55. 
108. Un livre de M. Arturo Farinelli sur "La Vida es sueño" et "Le grenier asturien." BH, xvm, 207-213.

109. Vieux procédés ou engins de guerre. BH, xviI, 52.

\section{7}

110. Appendices à la Chronique latine des Rois de Castille jusqu'en 1236. $\mathrm{BH}, \mathrm{xIx}, 101-115 ; 243-258$.

111. L'espionnage en Espagne au temps de la Reconquête. $\mathrm{BH}, \mathrm{xIx}, 259-$ 264.

112. Quelques lettres de Mariana et nouveaux documents sur son procès. $\mathrm{BH}, \mathrm{xIx}, 1-25$.

113. René Coste (nécrologie). $\mathrm{BH}, \mathrm{xIx}, 268-275$.

1918

114. Appendices à la Chronique latine des Rois de Castille jusqu'en 1236. $\mathrm{BH}, \mathrm{xx}, 27-35 ; 149-185$.

115. Un prospectus de Ferreras. $\mathrm{BH}, \mathrm{xx}, 257-263$.

R e s e $\tilde{n}$ a s :

116. Menéndez Pidal, Ramón. Cancionero de Romances impreso en Amberes sin año. Edición facsimile con una introducción por... Madrid, 1914. xlvii-275 pp. BH, xx, 202.

117. Paz, Julián. Archivo general de Simancas. Catálogo IV. Secretaria de Estado. (Capitulaciones con Francia y negociaciones diplomáticas de los embajadores de España en aquella corte, seguido de una serie cronológica de éstos.) Madrid, 1916. BH, $\mathrm{xx}, 203-204$.

118. Pereira Novaes, M. Collecção de manuscriptos ineditos agora dados a estampa. Anacrisis bistorial; Episcopologio. Porto, 4 tomos. 1912 1915. BH, xx, 194-197.

119. Prestage, D. Edgar. Francisco Manuel de Mello. Esbogo biographico. Coimbra, 1914. xxxv-614 pp. BH, xx, 138-140. 
120. Allá van Leys o mandan Reys. BH, XxI, 306.

121. A propos d'une édition récente de la Chronique d'Alphonse III. $\mathrm{BH}$, $\mathrm{xxI}, 1-8$.

122. Appendices à la Chronique latine des Rois de Castille jusqu'en 1236 (suite). BH, $\mathrm{xxI}, 173-192$.

123. La Chronique léonaise et les petites Annales de Castille. $\mathrm{BH}, \mathrm{xxI}$, 93-102.

124. L'Histoire de la Cava dans la chronique attribuée à Rasis. $\mathrm{BH}$, xxr, 297-306.

125. Le mouvement quaternaire dans les romances. $\mathrm{BH}, \mathrm{xxr}, 103-142$.

126. Recherches sur la Chronique latine des Rois de Castille. BH, xxI, 193-217; 276-281.

127. La "Semaine espagnole" de Paris. $\mathrm{BH}, \mathrm{xxI}, 245-246$.

Re $s$ e $\tilde{n}$ a $s:$

128. Mounier, André. Les faits et la doctrine économiques en Espagne sous Pbilippe V. Gerónimo de Ustariz (1670-1732). Bordeaux, 1900. 300 pp. BH, xxI, 247-249.

129. Paz y Melia, A. Series de los más importantes documentos del Archivo y Biblioteca del Excelentisimo Sr. Duque de Medinaceli elegidos y publicados a sus expensas. $1^{4}$ serie bistórica. Años $860-$ 1814. Madrid, 1915. BH, xxI, 78-82.

130. Pérez de Ayala, R. Política y toros. Ensayos. Madrid, Calleja, 1918. $\mathrm{BH}, \mathrm{xxr}, 172$.

131. Reynier, G. Le roman réaliste au XVIIe siècle. Paris, Haçhette, 1914. 395 pp. BH, xxI, 82-86.

132. Zugasti, J. A. Santa Teresa y la Compañia de Jesús. Estudio bistórico-crítico por el ... segunda edición, corregida y aumentada. Madrid, "Razón y Fe", 1914. BH, xxr, 311-318. 
1920

133. A propos des dernières publications sur Garcilaso de la Vega. BH, xxII, 234-255.

134. Les éditions des oeuvres de sainte Thérèse par La Fuente. $\mathrm{BH}$, xxil, 295-302.

135. L'enseignement de l'arabe à Bordeaux. BH, xxir, 117.

136. La Guerra de Granada et l'Austriada. BH, xxI, 149-159.

137. Le roman du P. Mariana. BH, xxII, 269-294.

138. Le témoignage de López de Ayala au sujet de D. Fadrique, frère de Pierre-le-Cruel. En: Hispania. Paris, v, Janvier-Mars, 70-76.

Res e $\tilde{n}$ a s :

139. Heaton, H. C. The Gloria d'Amor of Fra. Rocaberti. A catalan vision poem of the 15 th century edited with introduction, notes and glossary by... New York, Columbia University Press, 1916. xii-167 pp. BH, xxII, 52-59.

140. Miguélez, P. Catálogo de los Códices españoles de la Biblioteca del Escorial. I. Relaciones bistóricas. Madrid, Imprenta helénica, 1917. xlviii-363 pp. BH, xxII, 125-144.

141. Rennert, Hugo A. Bibliograpby of the dramatic works of Lope de Vega Carpio based upon the Catalogue of Jobn Rutter Chorley. (Extrait de la Revue Hispanique, Tome XXXIII, no. 83), 1915. $\mathrm{BH}, \mathrm{xxII}, 60-61$.

\section{$\begin{array}{llll}1 & 9 & 2 & 1\end{array}$}

142. Cervantes et les Frères Tharaud. BH, XXII, 57-59.

143. Fernán González dans la Chronique léonaise. BH, xxII, 1-14; 7794; 269-284.

$R$ e $s$ e $\tilde{n}$ a $s$ :

144. Analecta Montserratensia. Vol. I. Any 1917. Vol. II. Any 1918. $\mathrm{BH}, \mathrm{xxII}, 168-171$. 
145. Andrenio. Novelas y novelistas (Galdós, Baroja, Valle-Inclán, Ricardo León, Unamuno, Pérez de Ayala, Condesa de Pardo Bazán). Madrid, Calleja, 1918. 333 pp. BH, xxIII, 68-69.

146. Berwick y de Alba, Duque de. Contribución al estudio de la persona del III duque de Alba. Discursos leidos ante la R. Academia de la bistoria... el día 18 de mayo de 1919. Madrid, (1919). BH, xxiII, 66-68.

147. Boletín de la Biblioteca Menéndez Pelayo (año 1, 1919). BH, xxmr, 159-161.

148. Castro, Américo y Federico de Onís. Fueros leoneses de Zamora, Salamanca, Ledesma y Alba de Tormes. Edición y estudio de ... I. Textos. Madrid, 1916. 341 pp. BH, xxII, 239-243.

149. Deminuid, Mgr. Saint Jean de la Croix (1542-1591). Paris, Lecoffre, 1915 (2ème édition, 1916). 210 pp. BH, xxm, 346-351.

150. Domínguez Berrueta, J. Santa Teresa de Jesús y San Juan de la Cruz. (Bocetos psicológicos.) Madrid, Beltrán, 1915. 69 pp. BH, XxIIr, 346-351.

151. Frankowski, Eugeniusz. Hórreos y palafitos de la peninsula ibérica, por . . Madrid, Museo Nacional de Ciencias Naturales, 1918. 160 pp. BH, xxm, 250-254.

152. Guevara, Fr. Ant. de. Menosprecio de corte y alabanza de aldea. Edición $y$ notas de M. Martínez de Burgos. Madrid, La Lectura, 1915. BH, xxm, 243-244.

153. Hardy, G. et Paul Aurès. Les grandes étapes de l'bistoire du Maroc. Paris, Larosc, 1921. 135 pp. BH, xxmr, 355.

154. Lope de Vega. Teatro, tomo I. Peribáñez y el comendador de Ocaña. La estrella de Sevilla. El castigo sin venganza. La dama boba. Prólogo de Alfonso Reyes. Madrid, 1919. BH, XxIII, 244-245.

155. Llano Roza de Ampudia, A. de. El libro de Caravia por... Oviedo, Imprenta Gutenberg, 1919. 242 pp. BH, xxII, 76.

156. Monmarché, M. (et Pr. Ricard). Le Maroc, publié sous la direction de... 2ème édition. Paris, Hachette, 1921. xvi-396 pp. BH, xxıI, $353-354$. 
157. Orueta, R. de. Berruguete y su obra. Madrid, Calleja, 1917. BH, XxII, 144-146.

158. Pires de Lima, A. C. Tradições populares de Santo Tirso, separata da Revista Lusitana (Vol. XVIII). Porto, 1915 . BH, xxIII, 158-159.

159. Rodríguez Marín, Francisco. Cervantes, Novelas ejemplares. Edición y notas de... 2 vols. Madrid, "La lectura", 1914 y 1917. $\mathrm{BH}, \mathrm{xxII}, 153-158$.

160. Sánchez, Galo. Fueros castellanos de Soria y Alcalá de Henares. Estudio y edición de ... Madrid, 1919. xv-327 pp. BH, xxm, 239 243.

161. Toro Gisbert, Miguel de. Ortología castellana de nombres propios. Paris, 1914. 493 pp.; Americanismos. s. d. 287 pp.; Los nuevos derroteros del idioma. Paris, 1918. 376 pp. BH, xxII, 254-266.

\section{2}

162. Alpbonse le Noble et la Juive de Tolède. BH, xxrv, 289-306.

163. Fernán González dans la Chronique léonaise. BH, Xxıv, 193-197.

164. La question du latin et l'espagnol. $\mathrm{BH}, \mathrm{xxIV}, 84-86$.

165. Recherches sur les juifs espagnols et portugais à Bordeaux. Les vestiges de l'espagnol et du portugais dans le parler des juifs bordelais. (Supplément.) BH, xxIv, 203-224.

R e s e $\tilde{\mathbf{n}}$ a s :

166. Cour, Aug. La dynastie marocaine des Beni Wattas (1420-1554). Constantine, 1920.240 pp. BH, xxIv, 384-386.

167. García, E. Juan. Pérez Bayer y Salamanca. Datos para la biobibliografia del bebraista valenciano. Salamanca, Tipografía de Calatrava, 1918. 271 pp. BH, xxrv, 390-393.

168. Hermosilla, Diego de. Diálogo de la vida de los pages de Palacio, compuesto por Diego de Hermosilla. Edited with an introduction and notes by Donald Mackensie. Valladolid, 1916. BH, xxIv, $387-$ 388. 
169. Hésperis. Arcbives berbères et Bulletin de l'Institut des Hautes Études marocaines. Paris, Émile Larose, 1921. Nos. 1 and 2. BH, XxIv, 191.

170. Ibarra y Rodríguez, Ed. Documentos de asunto económico correspondientes al reinado de los Reyes Católicos (1475-1516). Fasc. I. preparado bajo la dirección de... Tomo I. Madrid, Tipografía de la Revista de Archivos, 1917. BH, xxiv, 386-387.

171. Laurencín, Marqués de. Los almirantes de Aragón. Datos para su cronología... Madrid, 1919. BH, xxIv, 191.

172. Marvaud, A. L'action économique française en Espagne. Paris, 1922. 91 pp. BH, xxrv, 288.

173. Morley, S. Griswold. Spanish bumor in Story and Essay, a book of selections for class reading. Chosen and edited with exercises, notes and vocabulary by ... Boston, Ginn \& Co., 1921. BH, xxrv, 396.

174. Sánchez Cantón, F. J. Don Juan Manuel. El Conde Lucanor. Prólogo y notas de... Madrid, Calleja, 1920. BH, xxIv, 167-173.

175. Solalinde, Antonio G. Calila y Dimna. Fábulas. Antigua versión castellana. Prólogo y vocabulario de ... Madrid, 1917. BH, xxiv, $167-173$.

175 a. Soldevia, Ferran. Pere II et Gran. El desafiament am Carles d'Anjou. Extret dels Estudis Universitaris Catalans. Barcelona, 1919, 54 pp. En: Revue Historique de Bordeaux, xv, 316-318.

\section{$\begin{array}{llll}1 & 9 & 2 & 3\end{array}$}

176. Un mémoire sur Saint Jean de la Croix. $\mathrm{BH}, \mathrm{xxv}, 265-266$.

177. Une mission médicale à Bordeaux. BH, xxv, 264-265.

178. M. Rafael Altamira, docteur "bonoris causa." BH, xxv, 264.

179. Rapport sur les concours d'agrégation d'espagnol et de certificat d'aptitude à l'enseignement de la langue espagnole dans les lycées et collèges en 1922. BH, xxv, 266-276.

180. Recherches sur la Chronique latine des Rois de Castille. IV. Quelques manuscrits de l'Escorial. $\mathrm{BH}, \mathrm{xxv}, 97-107$. 
181. Sur les romances. "A la muerte del príncipe de Portugal." $\mathrm{BH}, \mathrm{xxv}$, 168-172.

182. Valeur littéraire du "Viaje entretenido." $\mathrm{BH}, \mathrm{xxv}, 198-211$.

183. Visites d'universitaires portugais à Bordeaux. BH, $\mathrm{xxv}, 261-264$.

$R$ e $s$ e $\tilde{n}$ a $s:$

184. Beltrán y Rózpide, Ricardo. Crónica del emperador Carlos V, compuesta por Alonso de Santa Cruz, su cosmógrafo mayor y publicada... por D. Ricardo Beltrán y Rózpide y D. Antonio Blázquez y Aguilera. Madrid, 1920. Tomo r. xii-541pp. BH, xxv, 286-287.

185. Boissonnade, E. Du nouveau sur la "Chanson de Roland." La genèse bistorique, le cadre géograpbique, le milieu, les personnages, la date et l'auteur du poème. Paris, Champion, 1923. vi-520 pp. BH, $\mathrm{xxv}, 399-410$.

186. Castro, Américo. Les grands romantiques espagnols. Introduction, traduction et notes $d$ '... Paris, La "Renaissance du livre", s. d. $\mathrm{BH}, \mathrm{xxv}, 91-96$.

187. Farinelli, A. Viajes por España y Portugal desde la edad media basta el siglo XX. Divagaciones bibliográficas... Madrid, Centro de estudios Históricos, 1921. 511 pp. BH, xxv, 300-301.

188. Hurtado, J. y J. de la Serna et A. González Palencia. Historia de la literatura española. Parte primera. Madrid, 1921 . vii-480 pp.; Parte segunda. Madrid, 1922. Pp. 481-812; Parte tercera, pp. 8131106. BH, xxv, 187-189.

189. Keniston, Hayward. List of works for the study of Hispanic-American bistory. (Hispanic Notes and Monograpbs. V.) New York, The Hispanic Society of America, 1920. xviii-451 pp. $\mathrm{BH}, \mathrm{xxv}$, 293-294.

190. López Martínez, C. La Santa Hermandad de los Reyes Católicos. Sevilla, Vilches, 1921. 156 pp. BH, xxv, 179-180.

191. Mérimée, Ernest. Le poème du Cid. Extraits, traduction, introduction et notes par... Paris, s. d. BH, xxv, 91-96. 
192. Martinenche, Ernest. La Célestine, tragi-comédie de Calixte et Mélibée. Introduction de ... Paris, Collection "Les cent chefs d'oeuvre étrangers", s. d. BH, xxv, 91-96.

193. Pires de Lima, A. C. O Livro das adivinbas. Porto, Livraria moderna, 1921. 108 pp. BH, xxv, 189.

194. Sánchez Albornoz, Claudio. La Curia regia portuguesa. Siglo XII y XIII. Madrid, Centro de Estudios históricos, 1920. 188 pp. BH, $\mathrm{xxv}, 277-278$.

195. Sánchez Alonso, B. Fuentes de la bistoria española. Ensayo de bibliografía sistemática de las monografias impresas que ilustran la bistoria politica nacional de España, excluidas sus relaciones con América. Madrid, 1919. $\mathrm{BH}, \mathrm{xxv}, 186$.

196. Wiener, Leo. Africa and the discovery of America. Vol. I. Philadelphia, Innes \& sons, 1920. $\mathrm{BH}, \mathrm{xxv}, 285$.

\section{4}

197. De codicibus aliquot ad bistoriam Hispaniae antiquae pertinentibus olimque ab Ambrosio de Morales adbibitis. Burdigalae, apud Féret, 1924. (Parte del trabajo se relaciona con el Codex ovetensis de Pelagio, obispo de Oviedo.)

198. M. Rafael Altamira à Bordeaux. BH, xxvr, 275-280.

199. La vida de la madre Teresa de Jesús escrita de su misma mano, con una aprobación del padre M. fr. Domingo Banes... New York, Stechert \& Co., 1924-1929. 3 vols. (Introducción firmada) : Georges Cirot.)

$R$ es e ñ a :

200. Bell, Aubrey F. G. Baltasar Gracián. Oxford, Oxford University Press, 1921. 62 pp. BH, xxvI, 393.

201. Guerra y Sánchez, R. Historia de Cuba. Tomo I. 1492-1555. Ha_ bana, Imprenta "El siglo XX", 1921. 413 pp. BH, ххиI, 392-393.

202. Lucas-Dubreton, J. L'Espagne au XVe siècle. Le Roi sauvage. Paris, Librairie Perrin, 1922. BH, xxvi, 391-392. 
203. Martínez Torner, E. Cancionero musical de la lírica popular asturiana. Madrid, Nieto y Cía., 1920. 278 pp. BH, xxvI, 393.

\section{5}

204. Note sur l'Atalaya "de l'Arcbiprêtre de Talavera." En: Homenaje ofrecido a Ramón Menéndez Pidal. Madrid, 1925. Tomo r, pp. 355369.

R e $s$ e ñ a $s$ :

205. Calcott, Frank. The supernatural in early Spanisb literature, studied in the works of the Court of Alfonso X, el Sabio. New York, Instituto de las Españas, 1923. 151 pp. BH, xxvir, 254-257.

206. Clásicos Castellanos. Berceo. I. Milagros de Nuestra Señora, edición y notas de A. G. Solalinde. Madrid, "La lectura", 1922, tomo 44. $\mathrm{BH}, \mathrm{xxvII}, 355-357$.

207. Clásicos Castellanos. Tirso de Molina. Obras. I. Segunda edición, muy renovada, por Américo Castro. Madrid, "La lectura", 1922. Tomo 2; Moreto, Teatro, edición y notas de Narciso Alonso Cortés, tomo 32, $2^{\text {a }}$ edición. Madrid, 1922; Francisco de Rojas, Teatro, prólogo y notas de Federico Ruiz Morcuende, tomo $35 . \mathrm{BH}$, xxvm, 176-186.

208. Cortés, N. Alonso. El teatro en Valladolid. Madrid, "Revista de Archivos", 1923. 431 pp. BH, xxvII, 363-365.

209. Crawford, J. P. Wickersham. Spanish Drama before Lope de Vega by ... Philadelphia, University of Pennsylvania Press, 1922. 198 pp. (Publications of the University of Pennsylvania. Extra series in romanic Languages and literatures. No. 7.) BH, xxvm, 358-361.

210. Ezguerra del Bayo, J. Catálogo de las miniaturas y pequeños retratos pertenecientes al Excelentisimo Sr. Duque de Berwick $y$ de Alba. Madrid, Rivadeneyra, 1924. BH, xxvI, 186-187.

211. Fitzmaurice-Kelly, J. Antonio Pérez. London, Oxford University Press, 1922. x-170 pp. (Hispanic Notes \& Monographs. Essays, studies and brief biographies issued by the Hispanic Spciety of America. VI.) $\mathrm{BH}$, xxvir, 93. 
212. Fitzmaurice-Kelly, J. Gescbichte der Spanische Literatur übersetzt von Elisabeth Vischer, berausgegeben von Adalbert Hämel. Heidelberg, Carl Winters Universitätsbuchhandlung, 1925. BH, xxvIr, 285.

213. Hendrix, W. Samuel. Some native comic types in the early Spanish drama. (The Obio State University Bulletin, Sept. 15, 1924. Columbus, Ohio. Contribution in language and literatures No. 1.) $\mathrm{BH}, \mathrm{xxvII}, 361-362$.

214. Hoornaert, R. Sainte Thérèse écrivain. Son milieu, ses facultés, son oeuvre. Lille, Desclée, 1922. xii-652 pp. (Université de Louvain. Recueil de travaux publiés par les membres des conférences d'bistoire et de philologie.) BH, xxvr, 366-367.

215. Lonchay, Henry et Joseph Cuvelier. Correspondance de la Cour d'Espagne sur les affaires des Pays-Bas aux XVIIe siècle. Tome premier. Précis de la correspondence de Philippe III (1598-1621). Bruxelles, Kiessling, 1923. xvi-601 pp. BH, xxvII, 168-170.

216. Marden, C. Carroll, ed. Libro de Apolonio, an old Spanish Poem, edited by C. Carroll Marden. Part. II. Grammar, Notes and vocabulary. Princeton, Princeton University Press, 1922. 192 pp. (Elliott Monographs in the Romance languages $E$ literatures.) $\mathrm{BH}$, xxvI, $253-254$.

217. Menéndez Pidal, Ramón. Poesia juglaresca y juglares. Aspectos de la historia literaria y cultural de España. Madrid, Centro de Estudios Históricos, 1924. 488 pp. BH, xxvi, $350-355$.

218. Mérimée, Henri. Théâtre espagnol, t. I. Encina, Torres Nabarro, Lope de Rueda, Lope de Vega. Introduction et notes par... Paris, "La Renaissance du Livre." s. d. 227 pp. BH, xxviI, 366.

219. Peers, E. Allison. Spanish Mysticism. A preliminary survey. London, Methuen, 1924. xi-277 pp. BH, xxvIr, 367-369.

220. Ricard, P. Pour comprendre l'art musulman dans l'Afrique $d u$ Nord et en Espagne. (Bibliothèque du tourisme publiée sous la direction de Marcel Monmarché.) Paris, Hachette, s. d. BH, xxvII, 257-258. 
221. Shaffer Jack, W. The early entremés in Spain; the rise of a dramatic form. Philadelphia, University of Pennsylvania, 1923. $136 \mathrm{pp}$. (Publications of the University of Pennsylvania. Series in Romanic Languages and literatures, No. 8.) $\mathrm{BH}, \mathrm{xxvn,} 362-363$.

222. Teatro antiguo español, textos y estudios. III. Luis Vélez de Guevara. El Rey en su imaginación, publicada por J. Gómez Ocerín. Madrid, 1920. IV. Lope de Vega, El cuerdo loco, publicada por José F. Montesinos, 1922. V. Lope de Vega, La Corona merecida, publicada por José F. Montesinos, 1923. BH, xxvIr, 170-176.

\section{6}

223. Anecdotes ou légendes sur l'époque d'Alpbonse VIII. BH, xxmm, 246-259.

223 a. Discours prononcé au cimetière de la Chartrense, le 4 janvier 1926 au cours des obsèques de M. J. A. Bruiails. En: Revue Historique de Bordeaux, $\mathrm{xIx}, 7-11$.

224. L’bommage à D. Ramón Menéndez Pidal. BH, xxvm, 179.

225. Notes complémentaires sur l'Atalaya de l'archiprêtre de Talavera. $\mathrm{BH}$, xxvir, núm. 2, 140-154.

226. Roscidae valles. BH, xxvin, 375-378. (Sobre un artículo de M. Saroihandy sobre La légende de Ronceveaux, publicado en homenaje ofrecido a Menéndez Pidal).

227. Valeur littéraire des nouvelles de Lope de Vega. BH, xxvm, 321355.

R e s e $\tilde{n}$ a s :

228. Adams, N. B. The romantic dramas of Garcia Gutierrez. New York, Instituto de las Españas en los Estados Unidos, 1922. 149 pp. BH, xxvir, 392-393.

229. Amadé, Jean. Bibliograpbie critique pour l'étude des origines et des premières manifestations de la Renaissance littéraire en Catalogne au XIXe siècle. Toulouse, Privat, 1924. 88 pp. BH, xxvIr, $197-$ 200. 
230. Artigas, M. Don Luis de Góngora. Biografía y estudio critico por... Madrid, "Revista de Archivos", 1925. 492 pp. BH, xxvir, 297-298.

231. Barja, César. Literatura española. Libros y autores clásicos. 1923; Libros $y$ autores modernos. 1924. BH, xxvIr, 193-196.

232. Bell, Aubrey F. G. Francisco Sánchez el Brocense. London, Oxford University Press, 1925. xï-166 pp. BH, xxvil, 188-189.

233. Bell, Aubrey F. G. Juan Ginés de Sepúlveda. London, Oxford University Press, 1925. xii-108 pp. BH, xxvir, 389-390.

234. Berwick y de Alba, Duque de. Biblia traducida del bebreo al castellano por Rabi Mosé Arragel de Guadalfajara (1422-1433 ?) y publicada por... Tomo I, 1920; Tomo II, 1922. Madrid, Imprenta artística, xxi-849 and 933 pp. $\mathrm{BH}$, xxvirl, $95-99$.

235. Bohigas-Balaguer, R. Los textos españoles y gallego-portugueses de la Demanda del Santo Grial. Madrid, Revista de Filología Española, 1925. 151 pp. BH, xxvm, 383.

236. Burnam, John. Paleograpbia iberica. Fac-similés de manuscrits espagnols et Portugais (IX-XVe siècle), avec notices et iranscriptions. Paris, Honoré Champion, 1920 and 1925. 2 fascicules. BH, XxvII, 90-92.

237. Castro Guisasola, F. Observaciones sobre las fuentes literarias de la "Celestina." Madrid, Revista de Filología Española, 1924. BH, xxviII, 288-289.

238. García de Diego, V. Contribución al Diccionario bispánico etimológico. Madrid, Revista de Filología española, 1926. BH, xxvIr, 92-94.

239. Gracián, Baltasar. Pages caractéristiques, précedées d'une étude critique par A. Rouveyre. Traduction par V. Bouillier. Paris, Mercure de France, 1925. 322 pp. BH, xxvir, 105-108.

240. Historia da colonizaçăo portuguesa do Brazil. Vol. I. Rio de Janeiro, Sociedade editora, 1921. BH, xxvm, 103-104.

241. King, Georgiana G. The way of Saint James. 3 vols. New York, G. P. Putnam \& sons, 1920. BH, xxvir, 94-95. 
242. Krepinsky, M. Inflexión de las vocales en español, traducción y notas de Vicente Garcia de Diego. Madrid, (1926.) BH, xxvm, 92-94.

243. Krüger, Fritz. El dialecto de San Ciprián de Sanabria. Monografia leonesa por... Madrid, 1923. BH, xxvir, 92-94.

244. Menéndez Pidal, Ramón. El rey Rodrigo en la literatura. Madrid, "Revista de Archivos, Bibliotecas y Museos", 1924-1925. 247 pp. $\mathrm{BH}, \mathrm{xxvII}, 381-382$.

245. Pedrell, F. et H. Anglés. Els Madrigals $i$ la Missa de difunts d'En Brudieu, transcripcio $i$ notes bistoriques $i$ critiques por ... Barcelona, Institut d'Estudis Catalans, 1921. 246 pp. BH, xxvm, 383386.

246. Pietsch, Karl. Spanish Grail fragments. El libro de Josep Abarimatia, la Estoria de Merlin, Lancarote. Edited from the unique manuscript by ... I. Texts; II, Commentary. Chicago, The University of Chicago Press, 1924-1925. BH, xxvin, 186-188.

247. Salado Alvarez, V. Méjico peregrino; mejicanismos supervivientes en el inglés de Norte-América. Méjico, Talleres gráficos del Museo Nacional de Arqueología, 1924. 173 pp. BH, xxvin, 197.

248. Sảnchez, Galo. Libro de los fueros de Castilla, publicado por... Barcelona, Universidad de Barcelona, 1924. xvi-167 pp. BH, xxvrn, 382.

249. Universidad de Valladolid. Conferencias del curso, 1923-1924. Sere primera. BH, xxvmr, 202-203.

250. Ureña, R. de y A. Bonilla. Obras del maestro Jacobo de las Leyes, jurisconsulto del siglo XIII, publicadas por... Madrid, 1925. xxv411 pp. BH, xxviri, 182-184.

251. Withers, A. M. The sources of the poetry of Gutierre de Cetina. Philadelphia, University of Pennsylvania Press, 1923. 91 pp. BH, xxvin, 104-105.

252. Anecdotes ou légendes sur l'époque d'Alphonse VIII (suite). BH, XXIx, 145-173;241-254;337-350. 
253. Nebrija. Gramática de la lengua castellana (Salamanca, 1492). Muestra de la Istoria de las Antigüedades de España; reglas de orthographia en la lengua castellana, edited with an introduction and notes, by Ignacio González-Llubera. Oxford University Press, 1926. Ixii-272 pp. BH, xxIx, 318-320.

254. Sur le manuscrit portugais de la Cbronique générale "Port. 4" de la Bibliothèque Nationale de Paris. BH, xxxx, 199-210.

Res e ñ a s :

255. Anibal, C. E. Mira de Amescua. I. El arpa de David. Introduction and critical text; II. Lisardo, bis pseudonym. Columbus, Ohio State University Studies. Vol. II. (1925). 201 pp. BH, xxIx, 136-137.

256. Barja, César. En torno al lirismo gallego del siglo XIX. Northampton, Mass., Smith College; Paris, Champion, 1926. viii-149 pp. BH, Xxox, 227-228.

257. Bell, Aubrey F. G. Luis de Leon (A study of the Spanish rendissance). Oxford, Clarendon Press, 1925. 395. pp. BH, xxix, 128129.

258. Castro, Américo. El pensamiento de Cervantes. Madrid, Hernando, 1925. 408 pp. BH, xxIx, 129-136.

259. Cortés, N. Alonso. Zorrilla, poesias. Edición y notas de .. Madrid, Ediciones de "La Lectura", 1925. xx-290 pp. BH, xxix, 138-140.

260. Lope de Vega's. "El Castigo del Discreto", together with a study of conjugal bonor in bis theatre by W. Fichier. New York, Columbia University Press, 1925. 283 pp. BH, xxIx, 426-427.

261. Miguélez, P. Catálogo de los Códices españoles de la Biblioteca del Escorial. II. Relaciones bistóricas. Madrid, Talleres Voluntad, 1925. x-368 pp. BH, xxIx, 214-218.

262. Montesinos, J. F. Lope de Vega. El Marqués de las Navas. (Teatro antiguo español. Textos y estudios. VI.) Madrid, 1925. 213 pp. $\mathrm{BH}, \mathrm{xxIx}, 218-221$.

263. Northup, G. Tyler. An introduction to Spanish literature. Chicago, The University of Chicago Press, 1925. $x-473$ pp. BH, xxix, $232-$ 234. 
264. Serrano, D. Luciano. Cartulario de San Pedro de Arlanza, antiguo monasterio benedictino. Madrid, Centro de Estudios Históricos, 1925. xvi-299 pp. BH, xxrx, 126-128.

265. Sunyol, Dom Gregori Ma. Introducció a la Paleografia musical Gregoriana. Monje de Montserrat, 1925. 410 pp. BH, xxrx, 126.

266. Tiscornia, Eleuterio F. "Martin Fierro" comentado y anotado. Tomo I. Texto, notas y vocabulario. Buenos Aires, Coni, 1925. xx$501 \mathrm{pp} . \mathrm{BH}, \mathrm{xxx}, 225-227$.

267. Williams, E. Bucher. The life and dramatic works of Gertrudis Gómez de Avellaneda. Philadelphia, (University of Pennsylvania. Series in Romanic Languages and Literatures, no. 11), 1924. 116 pp. BH, xxix, 224-225.

\section{8}

268. Anecdotes ou légendes sur l'époque d'Alphonse VIII. $\mathrm{BH}, \mathrm{xxx}, 1-$ 70.

269. Deux notes sur les rapports entre romances et chroniques. BH, $\mathrm{XXx}$, 250-255.

270. Sur le "Fernán González." BH, xxx, 113-146.

Reseñ as :

271. Anuario de Historia del Derecho español. Tomo m. Madrid, Centro de Estudios Históricos, 1926. 600 pp. BH, xxx, 88-93.

272. Berwick y de Alba, Duque de. El Mariscal de Berwick, bosquejo biográfico por... Madrid, 1925. 538 pp. BH, xxx, 97.

273. Castro, Guillén de. La tragedia por los Celos. Comedia famosa de D. Guillén de Castro, edited after a 17 th century suelia, with an introduction, variants and notes by Hymen Alpern. Paris, Champion, 1926. 150 pp. BH, xxx, 96-97.

274. Corbière, A. S. Juan Eugenio Hartzenbusch and the French theater. Philadelphia, University of Pennsylvania Press, 1927. 95 pp. $\mathrm{BH}, \mathrm{xxx}, 358-359$.

275. Deferrari, Harry Austin. The sentimental Moor in Spanish titerature before 1600. Philadelphia, University of Pennsylvania Press, 1927. 84 pp. BH, xxx, 262-268. 
276. Entwistle, William J. The Artburian legend in the Literature of the Spanish Peninsula. London, J. M. Dent, 1925. vii-271 pp. BH, $\mathrm{xxx}, 85-88$.

277. Grossmann, R. Das ausländische Sprachgut im Sponischen des Rio de la Plata. Ein Beitrag zum Problem der argentinischen Nationalsprache von... Hamburg, 1926. 224 pp. BH, xxx, 356.

278. Ibarra, Eduardo. España bajo los Austrias. Barcelona, Editorial Labor, (1928). 380 pp. BH, xxx, 187-188.

279. Mapes, Erwin. L'influence française dans l'oeuvre de Rubén Dario. Paris, Champion, 1925. vii-183 pp. BH, xxx, 276-281.

280. Mayer, Ernesto. El antiguo derecho de obligaciones español según sus rasgos fundamentales. Barcelona, Librería Bosch, 1926. 309 pp. $\mathrm{BH}, \mathrm{xxx}, 334-335$.

281. Peers, E. Allison. Studies of the Spanish Mystics. Vol. I. London, The Sheldon Press, 1927. xvii-471 pp. BH, xxx, 339-347.

282. Valera, Mosén Diego de. Crónica de los Reyes Católicos, edición y estudio por Juan de Carriazo. Madrid, Revista de Filología española, 1927. cliv-314 pp. BH, xxx, 258-260.

\section{9}

283. A propos de la nouvelle de l'Abencerraje. BH, xxxı, no. 2, 131-138.

284. "El celoso extremeño" et l'Histoire de Floire et Blanceflor. BH, XXXI, 138-143.

285. La Chronique de D. Pedro Fernández de Velasco. BH, xxxr, 331339.

286. Encore les "Maris jaloux" de Cervantes. BH, xxxI, 339-346.

287. Gloses sur les "Maris jaloux" de Cervantes. BH, xxxI, 1-74.

288. Inauguration de l'Hôtel de l'Institut d'Etudes bispaniques de Paris. $\mathrm{BH}, \mathrm{xxxr}, 271-273$.

R e s e ñ a $\mathrm{s}$ :

289. Anuario de Historia del Derecho español. Tomo IV. (Madrid), Centro de Estudios Históricos, 1927. 532 pp. BH, XxxI, 275-276. 
290. Ballester y Castell, R. Las fuentes narrativas de la Historia de España duranie la Edad Moderna (1474-1808). Fasc. I. Los Reyes Católicos, Carlos I, Felipe II. Valladolid, 1927. 204 pp. BH, xxx, 367-368.

291. Ballesteros, Mercedes Gaibrois de. Sancho IV de Casillla. Tomo I. Madrid, 1922. xiv-240-ccii pp.; Tomo II, Madrid, Talleres Voluntad, 1928. 400 pp.; Tomo II, 1928, 520 pp. BH, xxxr, 156159.

292. Bullón y Fernández, Eloy. Un colaborador de los Reyes Católicos. El Dr. Palacios Rubios y sus obras por... Madrid, V. Suárez, 1927. xi-400 pp. BH, xxxI, 280-282.

293. Cuvelier, J. et J. Lefèvre. Correspondence de la cour d'Espagne sur les affaires des Pays-Bas au XVIIe siècle, recueil commencé par Henry Lonchay, et continué par... avec la collaboration de... Tomo II. Précis de la correspondence de Pbilippe IV avec linfante Isabelle (1621-1633). Bruxelles, Kiessling, 1927. xiv-799 pp. BH, $\mathrm{xxxI}, 171-172$.

294. Entwistle, W. J. The "Cantar de gesta" of Bernardo del Carpio. (From The Modern Language Review, xxm, núms. 3 and 4, 1928.) $\mathrm{BH}, \mathrm{xxxI}$, no. $4,354-355$.

295. Farinelli, Arturo. Italia e Spagna. Letteraíura moderne. Studi diretti da ... Torino, Fratelli Bocca, 1929. Tomo. I. xi-442 pp.; Tomo II. 462 pp. BH, $\mathrm{xxxI}, 282-283$.

296. Groult, P. Les Mystiques des Pays-Bas et la littérature espagnole du XVIe siècle. Louvain, 1927. 288 pp. BH, xxxr, 80-83.

297. Marden, C. Carroll. Cuatro poemas de Berceo (Milagros de la Iglesia robada $y$ de Teófilo, $y$ Vides de Sancta Oria $y d e$ San Millán). Nuevo manuscrito de la Real Academia Española. Edicion de C. Marden. Madrid, Hernando, 1928. 109 pp. BH, XXXX, 362-363.

298. Matulka, Bárbara. The Cid as a courlly Hero: From the Amadis to Comeille. New York, Columbia University Press, 1923. 54 pp. BH, XXXI, 366-367.

299. Menéndez Pida1, Ramón. La España del Cid. T'omo 1 . Madrid, Editorial Plutarco, 1929. iv -450 pp. BH, $\mathrm{xxxI}, 356-361$. 
300. Menéndez Pidal, Ramón. Flor nueva de romances viejos que recogió de la tradición antigua y moderna. 1928. 294 pp. $\mathrm{BH}, \mathrm{xxx}_{\text {, }}$ 160-162.

301. Ramos y Loscertales, J. Ma. Fuero de Jaca (última redacción) publicado por... Barcelona, Bosch, 1927. xliv-138 pp. BH, xxxi, 276-277.

302. Ribera y Tarragó, Julián. Disertaciones y Opísculos. Edición colectiva que en su jubilación del profesorado le of recen sus discipulos y amigos (1887-1927), con una introducción de Miguel Asin Palacios. Madrid, Jmprenta Mestre, 1928. 2 tomos. cxvi-637 y 796 pp. BH, xxxr, 349-354.

303. Sánchez Alonso, B. Fuentes de la bistoria española e bispanoamericana. Segunda edición, revisada y ampliada. 2 Vols. Madrid, 1927. xvi-633 y 468 pp. BH, xxxr, 371-373.

304. Switzer, Rebecca. The Ciceroniane Style in Fr. Luis de Granada. New York, Instituto de las Españas en los Estados Unidos, 1927. vi-159 pp. BH, xxxr, 167-170.

$$
1930
$$

305. De auctoribus ab Ambrosio de Morales adbibitis ad scribendam bis. toriam, pracsertim de Sebastiano, Sampiro, Isidoro "el de Beja." Madrid, 1930. 17 pp. (Extracto del Homenaje a Bonilla y Sasa Martin, publicado por la Facaltad de Filosofía y Letras de la Universidad Central. Tomo II, pp. 135-151.)

306. L'bistoire du comte Fernán González dans le manuscrit portugais de Paris. BH, Xxxin, 16-46.

307. Notes sur les "Juifs Portugais" de Bordeaux. En: Miscelanea de Estudos em Honra de D. Carolina Michaelis de Vasconcellos. Coimbra, Imprensa da Universidade, 1930.15 pp.

308. Nouvelles observations sur "Ser" et "Estar." En: Todd Memorial Volumes; philological studies. 1930. Vol. x, 91-121.

R e s e ñ a :

309. Asin, J. Oliver. Origen árabe de rebato, arrobda y sus bomónimos. Contribución al estudio de la bistoria medieval de la táctica mi- 
litar y de su léxico peninsular. Madrid, 1928. 110 pp. BH, xxxir, 274-276.

310. Baer, Fritz. Die Jïden in Cbristlicben Spanien. Evester Teil. Urkunden und Registen. I. Aragonien und Navarra. Berlin, Akademie Verlag, 1928. xxvii-1175 pp. BH, xxxII, 281-282.

311. Cano, Juan. La poética de Luzan. Toronto, The University of Toronto Press, 1928. 141 pp. BH, xxxII, 188-189.

312. Castro, Américo. Santa Teresa y otros ensayos. Historia nueva. Madrid, Central de ediciones y publicaciones, 1930. 279 pp. BH, xxxM, 81-82.

313. Castro, Américo, Agustín Millares Carlo y A. J. Battistessa. Biblia medieval romanceada seguin los manuscritos escurialenses $I-j-3$, I-j-8 y $I-j-6$. Pentatenco. Buenos Aires, Facultad de Filosofia y Letras, 1927. BH, xxxm, 79.

314. Dam, C. F. Adolfo van. El castigo sin venganza, tragedia de Frey Lope Félix de Vega Carpio. Edición conforme al monuscrito antógrafo de la Ticknor Library de Boston publicada con las variantes de los impresos, un estudio preliminar de la obra, notas al texto $y$ tres facsimiles, por... Groninga, P. Noordhoff, 1928. 414 pp. $\mathrm{BH}, \mathrm{xxxII}, 286-288$.

315. Douglass, Philip Earle. The Comedia Ypolita, edited with introduction and notes. Philadelphia, 1929. 98 pp. BH, xxxI, 420-421.

316. Gallardo, Bartolomé José. Obras escogidas de D. Bartolomé José Gallardo. Prólogo, edición y notas de Pedro Sáinz y Rodriguez. Madrid, 1928. 2 vols. xxxvi-267 y 324 pp. BH, xxxI, 189-190.

317. García Gómez, E. Un texto árabe de la leyenda de Alejandro según el manuscrito árabe XXVII de la Biblioteca de la Junta para ampliación de estudios. Edición, traducción espanola $y$ estudio preliminar... Madrid, 1929. clxiv-108-178 pp. BH, xxxII, 272274.

318. González Palencia, Angel. Historia de la literatura arábigo-española. Barcelona-Buenos Aires, Editorial Labor, 1928. 356 pp. BH, xxxIx, $75-77$. 
319. Green, Otis Howard. The life and works of Lupercio Leonardo de Argensola. Philadelphia, University of Pennsylvania Press, 1927. 203 pp. BH, xxxIr, 186-188.

320. Horne, J. Van. El Bernardo of Bernardo de Balbuena. A study of the Poem with particular attention to its relation to the Epics of Boiardo and Ariosto and to its significance in the Spanish Renaissance. [Chicago], 1927. 182 pp. BH, xxxi, 284-286.

321. Menéndez Pidal, Ramón. Orígenes del español. Estado lingiústico de la peninsula ibérica basta el siglo XI. Segunda edición, corregida y adicionada. Tomo 1. Madrid, Hernando, 1929. xii-591 pp. BH, XXXII, 268-269.

322. Montesinos, José F. Teatro antiguo español. Textos y estudios. VIII. Lope de Vega, El Cordobés valeroso Pedro carbonero, edición de... Madrid, Centro de Estudios Históricos, 1929. 213 pp. BH, xxxI, 421-424.

323. Montesinos, José F. Lope de Vega. Poesías líricas. Edición, prólogo y notas de... Madrid, Ediciones de "La Lectura" (vols. 68 y 75), 1926 y 1927.296 y 300 pp. BH, xxxII, 83.

324. Peers, E. Allison. Ramon Lull. A biograpby. New York and Toronto, The MacMillan Co., 1929. xvii-454 pp. BH, xxxII, 277-281.

325. Primicerio, Elena. La Historia del Abencervaje y los Romances de Granada. Introduzzione, revisione et note di . . Napoli, Fr. Giannini, 1929. 142 pp. BH, xxxII, 282-284.

326. Rey, Ct. García. El Deán don Diego de Castilla y la reconstrucción de Santo Domingo de Toledo. Toledo, A. Medina, 1928. 147 pp. BH, xxxII, 82-83.

327. Scudéry, Georges de. Le prince déguisé republished with and introduction by Barbara Matulka. New York, Institute of French Studies, 1929. 102 pp. BH, xxxm, 425-426.

328. Serrano, D. Luciano. Cartulario de Monasterio de Vega, con documentos de San Pelayo y Vega de Oviedo. Madrid, Junta para ampliación de estudios, 1927. xxix-212 pp. BH, xxxr, 78-79.

329. Vives, D. José. Juan Fernández de Heredia, Gran Maestre de Rodas por ... Barcelona, Balmes, 1927.79 pp. BH, xxxr, 80-81. 
330. Le "Carmen Campidoctoris." BH, xxxiI, 144-149.

331. L'Hirondelle et les petits oiseaux dans "El conde Lucanor." $\mathrm{BH}$, XxxiIr, no. $2,140-143$.

332. Góngora et Musée. BH, xxxmi, 328-333.

333. Le rytbme du "Carmen Campidoctoris." BH, xxxIII, 247-252.

334. Sur le Fernán González. BH, xxxII, 104-115.

$\mathrm{R}$ e s e $\tilde{\mathbf{n}}$ as:

335. Artigas, M. y P. Sainz y Rodríguez. Epistolario de Valera y Menéndez Pelayo, publicado con una introducción y notas. Madrid, CIAP, 1930. 255 pp. BH, Xxxur, 275-277.

336. Berceo. Veintitrés milagros. Nuevo manuscrito de la Real Academia Española. Edición de... Madrid, Hernando, 1929. 103 pp. BH, xxxmr, 55-56.

337. Bullón, E. Miguel Servet y la geografia del Renacimiento. Segunda edición. Madrid, V. Suárez, 1929. 207 pp. BH, xxxmI, 62-64.

338. Farinelli, Arturo. Viajes por España y Portugal. Suplemento al volumen de las Divagaciones bibliográficas. Madrid, Centro de Estudios Históricos, 1930. 566 pp. BH, xxxm, 366.

339. Gandía, E. de. Del origen de los nombres y apellidos y de la ciencia genealógica. Buenos Aires, Editorial "La Facultad", 1931. 327 pp. BH, xxxII, 273-275.

340. Gandia, E. de. Historia crítica de los mitos de la Conquista americana. Madrid, 1929. 285 pp. BH, xxxm, 59-60.

341. Giulian, A. A. Martial and the Epigram in Spain in the 16 th and 17 ih centuries. Philadelphia, Univ. of Penn. Press, 1930. 117 pp. BH, xxxIII, 259-261.

342. González Palencia, A. Los mozárabes de Toledo en los siglos XII y XIII. Madrid, 1926-1930. 3 vols. viii-326, viii-598, xvi-462 pp. $\mathrm{BH}, \mathrm{xxxIIx}, 255-259$. 
343. Harlan, Marcel Margaret. Lope de Vega's El Desdén vengado, edited with introduction and notes. New York, Instituto de las Españas en los Estados Unidos, 1930. xlix-196 pp. BH, xxxm, 355356.

344. Louro, Estanco. O Livro de Alportel. Monografía de uma freguesia rural-concelbo. Lisboa, Livraria Sa da Costa, 1929. 477 pp. BH, xxxmi, 172-173.

345. Marañón, Gregorio. Ensayo biológico sobre Enrique IV de Casilla y su tiempo. Madrid, Mundo Latino, 1930. $182 \mathrm{pp} . \mathrm{BH}$, xxxm, 352-353.

346. Menéndez Pidal, Ramón. La España del Cid, tomo II (pages 4511006). Madrid, Editorial Plutarco, 1929. BH, xxxm, 47-52.

347. Meredith, J. A. Introito and Loa in the Spanish Drama of the 16th century. Philadelphia, University of Pennsylvania Press, 1928. BH, xxxII, 60-62.

348. Millé y Giménez, J. Sobre la génesis del Quijote. Cervantes, Lope de Vega, Góngora, el "Romancero general", el "Entremés de los Romances", etc. Primera edición. Barcelona, Casa editorial Araluce, (1930). 219 pp. BH, xxxm, 261-262.

349. Moldenhauer, G. Die Legende von Barlaam und Josaphat auf der iberischen balbinsel. Untersucbungen und Texte von... Halle, Max Niemeyer Verlag, 1929. 186-v-348 pp. BH, xxxu, 52-55.

350. Northup, G. T. El cuento de Trisian de Leonis edited from the unique manuscript Vatican 6428 by ... Chicago, The University of Chicago Press, 1928. 298 pp. BH, xxxm, 56-58.

351. Pastor, J. F. Las apologías de la lengua castellana en el siglo de oro. Seleccion y estudio por ... Madrid, 1929. xxx-188 pp. BH, xxxn, 163.

352. Pió, Príncipe. Documentos de mi archivo. La elección de Fernando IV, Rey de Romanos. Correspondencia del III marqués de Castel-Rodrigo Don Francisco de Moura durante el tiempo de su enbajada en Alemania (1648-1656). La publica según los originales que obran en el Archivo de sa casa... Madrid, 1929. xxviii-562 pp. $\mathrm{BH}, \mathrm{xxxII}, 67-69$. 
353. Pons, J. S. La littérature catalane en Roussillon au XVIIe et au XVIIIe siècle. L'Esprit provincial. Les Mystiques, les Goigs et le théâtre religieux. Toulouse, E. Privat, 1929. xxi-397 pp. BH, xxxm, 262-264.

354. Reparaz, Gonzalo de. La Constitución natural de España y las de papel (Mamual del perfecto constitucional español). Barcelona, 1928. 252 pp. BH, xxxm, 178-180.

355. Reparaz, Gonzalo de. Geografía y Politica. Veinticinco lecciones de bistoria naturalista. Barcelona, 1929. 277 pp. BH, xxxm, 178180.

356. Sargent, Cecilia Vennard. A study of the dramatic works of Cristóbal de Virués. New York, Instituto de las Españas en los Estados Unidos, 1930. ix-161 pp. BH, xxxII, 353-35s.

357. Serrano, D. L. Cartulario de San Vicente de Oviedo (781-1200). Madrid, Centro de Estudios Históricos, 1929. Ixiii-336 pp. BH, xxxirr, $45-47$.

358. Solenni, G. de. Lope de Vega's El Brasil restituido together with a study of patriotism in his theater. New York, Instituto de las Españas, 1929. cxli-159 pp. BH, xxxmI, 66-67.

359. Torres, M. El origen del sistema de "Iglesias propias." Madrid, "Revista de Archivos", 1929. 137 pp. BH, xxxiII, 44-45.

360. Wagner, Ch. Ph. El libro del Cauallero Zifar (El libro del Cavallero de Dios), edited from the three existant versions by... Part. I. Text. Ann Arbor, University of Michigan, 1929. xvi-532 pp. BH, xxxm, 58-59.

1932

361. Le "Compendio bistorial" de Garibay. BH, xxurv, 223-234.

362. J. Sarö̈bandy (necrologie). BH, xxxIv, 327-328.

363. Sur les romances "del Maestro de Calatrava." BH, xxxrv, 5-26. 
$R$ e $s$ eñ as :

364. Abúbequer de Tortosa. Lámpara de los Principes por... Traducción española de Maximiliano Alarcón. Tomo I. Madrid, 1930. Ixiii438 pp. Tomo Ir. Madrid, 1931.548 pp. BH, xxxrv, 168-170.

365. Bach y Rita, Pedro. The works of Pere Toroella a Catalan writer of the 15 th century. New York, Instituto de las Españas en los Estados Unidos, 1930. xx-332 pp. BH, xxxiv, 173-175.

366. Capellani, Andreae. De Amore ... Text llati amb la traducció Catalana del segle XIV. Introducció $i$ notes per Amadeu Pagès. Castilló de la Plana, 1930. xxxi-214-cxxi pp. BH, xxxiv, 170-173.

367. Castro, Américo. Cervantes. Paris, Editions Rieder, 1932. 80 pp. $\mathrm{BH}, \mathrm{xxxxy}, 268-271$.

368. Davids, J. A. De Orasio et sancto Augustino Priscillianistarum adversariis. Commentatio bistorica et philologica. Hagae, A. No. Govers, 1930. 301 pp. BH, xxxiv, 70-74.

369. Lonchay, H., J. Cuvilier, J. Lefèvre. Correspondence de la Cour d'Espagne sur les affaires des Pays-Bas aux XVIIe siècle. Tome III. Précis de la correspondence de Pbilippe IV (1633-1647). Bruxelles, Maurice Mamertin, 1930. xviii-743 pp. BH, xxxrv, 181-183.

370. Llorens, E. L. La negación en español antiguo, con referencias a otros idiomas. Madrid, Revista de Filología Española, 1929. 199 pp. $\mathrm{BH}, \mathrm{xxxIv}, 179-181$.

371. Matulka, Bárbara. The novels of Juan de Flores and their European diffusion. A study in comparative literature. New York University, Institute of French Studies, 1930. xvii-475 pp. BH, xxxrv, $175-179$.

372. Nykl, A. R. A book containing the Risala known as The Dove's neck-ring, about love and lovers, composed by Abu Mubammad Ali ibn-Hazan al-andalusi... translated from the unique MS. in the University of Leiden, edited by D. K. Petrof in 1914, by A. R. Nykl. Paris, Librairie Paul Genthner, 1931. cxxiv-224 pp. BH, xxxrv, 167-168. 
373. Paz, Julián. Catálogo de la colección de documentos inéditos para la bistoria de España. 2 tomos. Madrid, 1930-1931. xvii-728 y 874 pp. BH, xxxiv, 183-184.

374. Reparaz, (hijo), G. de. La época de los grandes descubrimientos españoles y portugueses. Barcelona, Labor, 1931.206 pp. BH, xxxrv, 263-265.

375. Sacramentado, P. Crisógono de Jesús. San Juan de la Cruz, su obra cientifica y su obra literaria, por... Tomo $I$, su obra cientifica. Tomo II, su obra literaria. Madrid, 1929. 499, 476 pp. BH, xxxiv, 336-34s.

376. Thomas, Lucien-Paul. Don Luis de Góngora y Argote. Introduction, traduction et notes par... Paris, La Renaissance du livre, 1932. 166 pp. BH, xxxiv, 271-273.

377. Wilson, Edward Meryon. The solitudes of Don Luis de Góngora $y$ Argote, translated into Englisb verse by ... Cambridge, 1932. n. p. BH, xxxiv, 271-273.

\section{3}

378. Le "Compendio bistorial" de Garibay. $\mathrm{BH}, \mathrm{xxxv}, 337-356$.

379. Desgastada-desgustada. $\mathrm{BH}, \mathrm{xxxv}, 298-299$.

Res e ñ $s$ :

380. Amezúa, A. de. Formación y elementos de la novela cortesana. Discursos leidos ante la R. Academia Española por... el dia 24 de febrero de 1929. Madrid, Tip. de Archivos, 1929. 152 pp. BH, $\mathrm{xxxv}, 459-460$.

381. Anspach, Eduard. Taionis et Isidori noua fragmenta et opera edidit et illustranit . . Madrid, Centro de Estudios Históricos, 1930. viii183 pp. BH, xxxv, 184-186.

382. Cossio, J. M. de. Obras escogidas de Salvador Jacinto Polo de Medina. Estudio, edición y notas de . . Madrid, "Los clásicos olvidados", 1931. 389 pp. BH, xxxv, 460-463.

383. Durán y Sampere, A. et Joseph Sanabre, edit. Llibre de les Solemnitats de Barcelona. Edició completa del MS. de l'Arxiu bistoric 
de la Ciutat. Vol. I. 1424-1546. Barcelona, 1930. xxiii-462 pp. BH, $\mathrm{xxxv}, 187-188$.

384. Figueiredo, Fidelino de. As duas Espanbas. Liçoes feitas no Instituto de Altos Estudos nos días 27, 29 e 30 de Janeiro de 1932 . . Coimbra, Imprensa da Universidade, 1932. 263 pp. BH, xxxv, 199-202.

385. Ford, J. D. M. editor. Letters of John III, King of Portugal, 15211557. The portuguese text edited with an introduction by... Cambridge, Harvard University Press, 1931. xxx-408 pp. BH, XXXV, 189-190.

386. Ford, J. D. M. and Ruth Lansing. Cervantes, a tentative bibliography of bis works and of the biograpbical and critical material concerning bim, prepared by ... Cambridge, Harvard University Press, 1931. xïi-239 pp. BH, xxxv, 193-194.

387. Horn, John van, editor. La grandeza mexicana de Bernardo de Balbuena. Editada según las primitivas ediciones de 1604, con uma introducción y con notas sobre las obras y los autores citados por Balbuena ... 1930. 176 pp. (University of Illinois Studies in Language and Literature.) $\mathrm{BH}, \mathrm{xxxv}, 192-193$.

388. Ibn Sabara, Joseph Benmeir. Llibre d'Ensenyaments delectables. (Sefer Xaaxuim.) Traducció d'Ignasi González-Lhibera. Barcelona, Editorial Alpha, 1931. xxv-194 pp. BH, xxxv, 186-187.

389. Lambert, Elie. L'art gotbique en Espagne aux XIIe et XIIIe siècles. Paris, Laurens, 1931. 314 pp. BH, xxxv, 70-76.

390. Leavitt, Sturgis E. The Estrella de Sevilla and Claramonte by... Cambridge, Harvard University Press, 1931. xii-111 pp. BH, xxxv, 463-464.

391. Mele, Eugenio, trad. Il libro dell Amico e dell'Amato di Ramon Lull. Versione, introduzione e commento di... Lanciano, 1932.

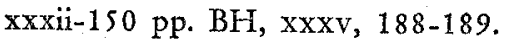

392. Paz, Julián. Catálogo de documentos españoles existentes en el Archivo del Ministerio de Negocios Extranjeros de Paris. Madrid, 1932. xxiii-402 pp. BH, xxxv, 464-466. 
393. Reparaz, Gonzalo de. Páginas turbias de historia de España. Páginas antiguas. (Segunda edición corregida y aumentada.) Barcelona, Ediciones Mentora, 1931. 2 vols. 282 pp. each. BH. xxxv, 78-83.

394. Ribelles Comín, José. Bibliografía de la lengua valenciana o sea catálogo razonado por orden alfabético de autores, de los libros, folletos, obras dramáticas, periódicos, coloquios, chistes, discursos, romances, alocuciones, cantares, gozos, etc. que escritos en lengua valenciana y bilingüe, ban visto la luz pública desde el establecimiento de la imprenta en España hasta nuestros dias. Tomo II. (Siglo XVI.) Madrid, Tipografía de la "Revista de Archivos", 1929. 649 pp. BH, xxxv, 76-78.

395. Wagner, M. L. et Max A. Luria. Caracteres generales del Judeoespañol de Oriente. 1930.120 pp.; A Study of the Monastir dialect of Judeo-Spanish. New York, 1930. 261 pp. BH, xxxv, 319-322.

\section{4}

396. Index onomastique et géographique de la Chronique léonaise. BH, xxxvi, 401-425.

397. "Per Deuia Alauae." BH, XxxvI, 88-93.

Reseñ a s :

398. Acevedo y Huelves, Bernardo, y Marcelino Fernández y Fernández. Archivo de tradiciones populares. III. Vocabulario del bable de Occidente. Madrid, Centro de Estudios Históricos, 1932. vii-242 pp. BH, xxxvi, 240-241.

399. Alfarabi. Catálogo de las ciencias, edi. y trad. castellana por A. González Palencia. Vol. II. Madrid, Imprenta de Estanislao Maestre, 1932. xix-176-184 pp. BH, xxxvi, 98-99.

400. Altamira, Rafael. Manual de bistoria de España. Madrid, M. Aguilar, 1934. 621 pp. BH, xxxvi, 510-514.

401. Asín Palacios, Miguel. Vidas de Santones andaluces. La "Epístola de la Santidad" de Ibn Arabi de Murcia. Madrid, Imprenta Maestre, 1933. 202 pp. BH, xxxvr, 99-103.

402. Atkinson, W. C. Spain, a brief bistory. London, Methuen and Co. Ltd., 1934. x-200 pp. BH, xxxvr, 380-381. 
403. Bertrand, L. Historia de España, trad. del francés por Luys Santa Marina. Barcelona, Sucesores de Jean Gili, 1933. 421 pp. BH, xxxvi, 377-380.

404. Carvajal, Micael de. Tragedia Josepbina, edited by Joseph E. Gillet. Princeton, Princeton University Press, 1932. 1xiv-205 pp. BH, XXxVI, 106-108.

405. Crece, San Giovanni della. Aforismi e Poessie, a cura di Don Giuseppe de Luca. Brescia, Morcelliana, 1933. 223 pp. BH, xxxvI, $108-$ 110.

406. Cuvielier, J. et J. Lefèvre. Correspondence de la cour d'Espagne sur les affaires des Pays-Bas aux XVIle siècle. Tome $\mathrm{IV}$. Précis de la correspondence de Pbilippe IV (1647-1665). Bruxelles, 1933. xv879 pp. BH, xxxvi, 527-530.

407. Farinelli, A.; G. M. Bertini, D. G. de Luca. Mistici di Spagna. Brescia, Morcelliana, 1933. cxix-177 pp. BH, xxxv1, 108-110.'

408. Fuero de Madrid. Publicaciones del Arcbivo de la Villa. Madrid, 1932. 73 folios. BH, XxxyI, 523-525.

409. González Palencia, Angel. Don Luis de Zúñiga y Avila, gentilbombre de Carlos V, por ... Madrid, Estanislao Maestre, 1932. 227 pp. BH, xxxvi, 230.

410. Guerra, J. C. Ilustraciones genealogicas de Garibay, referentes a solares vascos. San Sebastián, Nueva Editorial, 1933.239 pp. BH, xxxvr, 381-383.

411. Hauser, Henri. Peuples et civilisations. Histoire générale publiée sous la direction de ... La préponderance espagnole (1559-1660). Paris, Felix Alcan, 1933. 594 pp. BH, xxxvi, 231-234.

412. Lévi-Provençal, E. L'Espagne musulmane au Xe siècle. Institutions et vie sociale. Paris, Larose, 1932. 272 pp. BH, xxxvI, 95-98.

413. Nykl, A. R. El cancionero del Seih, nobilísimo visir, maravilla del tiempo. Madrid, Maestre, 1933. 1-xvi-466 pp. BH, xxxvI, 371-374.

414. Romera Navarro, M. Antologia de la literatura española desde los origenes basta principios del siglo XIX ... New York, D. C. Heath \& Co., 1933. v-427 pp. BH, xxxvr, 241-242. 
415. Toro, Gisbert M. de. L'évolution de la langue espagnole en Argentine; un fragmento del Diccionario general de la lengua española. Paris, Larousse, 1932. vi-201 pp. and x-82 pp. BH, xxxvi, 525-527.

416. Zarco Cuevas, F. J. Pintores españoles en San Lorenzo Real de El. Escorial; Pintores italianos en San Lorenzo el Real de El Escorial (1575-1613). Madrid, Academia de la Historia, 1932. xxxi-327 pp. and $\mathrm{xxx}-271$ pp. BH, xxxvi, 116-117.

\section{5}

417. A propos des Juifs portugais (with Joseph Benoliel). $\mathrm{BH}$, xxxvm, 487-489.

418. Le "Compendio historial" de Garibay. BH, xxxvm, 149-158.

419. Notes sur l'bistoriographie bispano-portugaise: I. La critique portugaise et Annius de Viterbe. BH, xxxvn, 454-459.

420. La topographie amourense du Marquis de Santillane. $\mathrm{BH}$, xxxvm, 392-395.

421. Une élégie latine du P. Mariana avec la réponse. (Extrait des Mélanges Paul Lammonier, 1935, pp. 369-376.)

Res e $\tilde{n}$ a $s:$

422. Albe, le Duc d'. Lettres familières de l'impératrice Eugénie, publiées par... Préface de Gabriel Hanotanx. Paris, Le Divan, 1935. 2 vols. xxv-227 and 245 pp. BH, xxxvIr, 523-524.

423. Asín Palacios, Miguel. La espiritudidad de Algazel y su sentido cristiano. Madrid-Granada, Publicaciones de las Escuelas de Estudios árabes de Madrid y Granada, 1934-1935. 2 vols. 532 y 566 pp. BH, xxxvII, 508-511.

424. Balseiro, José A. Novelistas españoles modernos. New York, The MacMillan Company, 1933. xxi-476 pp. BH, xxxvi, 119.

425. Cortés, N. Alonso. Artículos bistórico-literarios. Valladolid, Imprenta Castellana Montero Calvo, 1935. 224 pp. BH, xxxvI, 253254. 
426. Ford, J. D. M. and L. G. Moffatt, editors. Letters of the Court of Jobn III, King of Portugal. Cambridge, Harvard University Press, 1933. xix-169 pp. BH, xxxvir, 236.

427. Guinard, Paul. Madrid, l'Escorial et les anciennes residences royales. Paris, Librairie Renouard, 1935. 196 pp. BH, xxxvir, 246-247.

428. Hainsworth, G. Les "Novelas exemplares" de Cervantes en France au XVII siècle. Contribution à l'étude de la Nouvelle en France. Paris, Champion, 1933. 298 pp. BH, xxxvII, 238-240.

429. Isaza y Calderón, B. El retorno a la naturaleza. Los orígenes del tema $y$ sus direcciones fundamentales en la literatura española. Madrid, Bolaños y Aguilar, 1934. 260 pp. BH, xxxvir, 251-253.

430. Le Gentil, Georges. La littérature portugaise. Paris, A. Colin, 1925. 208 pp. BH, xxxvII, 527-530.

431. Marañón, Gregorio. Las ideas biológicas del Padre Feijóo. Madrid, Espasa-Calpe, 1934. 335 pp. BH, xxxvir, 114-115.

432. Martínez de la Rosa. Obras dramáticas. La viuda de Padilla, Aben Humeya, y la Conjuración de Venecia. Edición y notas de Jean Sarrailh. Madrid, Espasa-Calpe, 1933. 414 pp. (La Lectura, No. 107.) $\mathrm{BH}, \mathrm{xxxvm}, 115-116$.

433. Menéndez Pidal, Ramón. La leyenda de los Infantes de Lara. Madrid, 1934. xvi-490 pp.; Historia y Epopeya. Madrid, 1934. vi309 pp. BH, xxxvI, 403-407.

434. Millé y Giménez, Juan. La vida de Estebanillo González, bombre de buen bumor, compuesto por él mismo. Edición y notas de... Madrid, Espasa-Calpe, 1934. 2 vols. 262 y 270 pp. BH, xxxvIr, 407-408.

435. Morínigo, Marcos A. Hispanismos en el guaraní. Estudio sobre la penetración de la cultura española en la guarani, según se refleja en la lengua, bajo la dirección de Amado Alonso. Buenos Aires, 1931. 433 pp. BH, xxxvir, 116-118.

436. Paz, Julián. Catálogo de manuscritos de América existentes en la Biblioteca Nacional... Madrid, Tipografía de Archivos, 1933. viii727 pp. BH, xxxvII, 113. 
437. Pérez de Urbel, Fr. Justo. Los monjes españoles en la Edad Media. Madrid, Instituto de Valencia de Don Juan, 1933-1934. 2 tomos de 529 y 642 pp. BH, xxxvII, 501-508.

438. Ricard, Robert. La conquête spirituelle du Méxique. Essai sur i'Apostolat et les méthodes missionnaires des Ordres mendiants en Nouvelle Espagne de 1523-1524 à 1572. Paris, Institut d'Ethnologie, 1933. xix-404 pp. BH, xxxvII, 109-112.

$$
1936
$$

439. Le "cautivo" de Cervantes et Notre-Dame de Liesse. BH, xxxvm, 378-382.

440. Le "chant d'Altabiscar." BH, xxxvm, 65-67.

441. Le chemin de Compostelle, d'après Madoz et Morales. BH, xxxvm, $537-538$.

442. "Ladino" et "aljamiado." BH, xxxvur, 538-540.

443. Mariana jésuite. La jeunesse. $\mathrm{BH}$, xxxvII, 295-352.

444. Notes sur l'bistoriographie bispano-portugaise. BH, xxxvII, 417443.

R e $s$ e $\tilde{n}$ a $s$ :

445. Al Saqundi. Elogio del Islam español (Risala fi fadl al-Andalus). Traducción española por Emilio Garcia Gómez. Madrid, Imprenta Maestre, 1934. 123 pp: BH, xxxvmI, 86-87.

446. Alonso, Dámaso. Don Luis de Góngora. Obras mayores. I. Las Soledades nuevamente publicadas. Madrid, Ediciones del Arbol, 1935. 432 pp. BH, xxxviII, 397-399.

447. Amezúa A. de. Lope de Vega en sus cartas. Introducción al Epistolario de Lope de Vega Carpio que por acuerdo de la Academia Española publica... Madrid, Tipografía de Archivos, 1935. xiv-525 pp. BH, xxxvII, 401-404.

448. Andersson, Th. Carlos Maria Ocantos, argentine novelist. A study of indigenous French and Spanish elements in bis work. New Haven, Yale University Press, 1934. xii-136 pp. BH, xxxvin, 110-113. 
449. Cerny, Václav. Essai sur le Titanisme dans la poésie romantique occidentale entre 1815 et 1850. Prague, Aux Editions Orbis, 1935. 437 pp. BH, xxxvm, 235-241.

450. Lanz, L. Valenilla, C. Parra-Pérez, Ch. V. Aubrun. Bolivar, choix de lettres, discours et proclamations. Paris, Librairie Stock, 1936. 333 pp. BH, xxxvin, 235.

451. Long, Wasley Robertson, editor. La flor de las Ystorias de Orient by Hayton, prince of Gorigos, edited from the unique MS. Escorial Z-I-2, with introduction, bibliography and notes by . . Chicago, The University of Chicago Press, 1934. viii-223 pp. BH, xxxviII, 93-95.

452. Marasso, A. Rubén Dario y su creación poéíca. La Plata, Universidad de la Plata, 1934. xxvi-410 pp. BH, xxxvir, 107-110.

453. Menéndez Pidal, Ramón. Historia de España, dirigida por . . T. II. España romana (218 a de J. C. - 414 de J. C.) Madrid, EspasaCalpe, 1935. xl-811 pp. BH, xxxvir, 391-393.

454. Menéndez Pidal, R. et Varón Vallejo. Historia Troyana en prosa y verso, texto de hacia 1270, publicada por... con la colaboración de E. Varón Vallejo. Madrid, S. Aguirre, 1934. 1-227 pp. $\mathrm{BH}_{3}$ xxxvir, $91-93$.

455. Merriman, R. B. The rise of the Spanish Empire in the old World and the New. Vol. IV. Pbilip the Prudent. New York, The MacMillan Co., 1934. xxiv-780 pp. BH, xxxvmI, 98-102.

456. Montesinos, José F. Teatro antiguo español. Textos y estudios. VIII. Lope de Vega, Barlaan y Josafat. Madrid, Centro de Estudios Históricos, 1935. 311 pp. BH, xxxvII, 233-235.

457. Pagès, A. Les cobles de Jacme, Pere é Arnau March. La poessia lírica d'Abans d'Auzias Marcb. Introducció $i$ anotació d'Amadeu Pagès. Castelló de la Plana, 1934. 121 pp. BH, xxxvnI, 95-96.

458. Pareja Casañas, F. M. Libro de Ajedrez, de sus problemas y sutilezas, de autor árabe desconocido. Madrid, 1935. 2 tomos. viii-260 y 252 pp. BH, xxxvir, 393-394. 
459. Roza de Ampudia, A. de Llano. Pequeños anales de quince días. La Revolución en Asturias, octubre 1934. Oviedo, Talleres Altamirano, 1935.215 pp. BH, xxxvm, 114.

460. Thorndike, Lynn. A bistory of magic and experimental sciences. Vol. III and IV. Fourteenth and fifteenth centuries. New York, Columbia University Press, 1934. xxvi-827 and xviii-767 pp. BH, XxxvII, 96-98.

461. Willis, H. The debt of the Spanish Libro de Alexandre to the french Roman d'Alexandre. Princeton, Princeton University Press, 1935. vi-59 pp. BH, xxxvIII, 394-397.

462. Willis, R. S. The relationship of the Spanish "Libro de Alexandre" to the "Alexandreis" of Gautier de Chatillon; El Libro de Alexandre, Texis of the Paris and the Madrid manuscripts prepared with an introduction by . . Princeton, Princeton University Press, 1934. vi-94 and xl-461 pp. BH, xxxvm, 87-91.

\section{7}

463. La guerre de Troie dans le Libro de Alexandre. BH, xxxIx, 328338.

R e s e $\tilde{\mathbf{n}}$ a s :

464. Alonso, Dámaso. La lengua poética de Góngora (Primera parte). Madrid, S. Aguirre, 1935. 230 pp. BH, xxxIx, 64-68.

465. Bailly, Ch., Elise Richter, A. Alonso, R. Lida. El impresionismo en el lenguaje. Buenos Aires, Instituto de Filología, 1936.278 pp. BH, $\mathrm{XxXIX}, 415-416$.

466. Ballesteros, Mercedes Gaibrois de. Vidas memorables. Maria de Molina por ... Madrid, Espasa-Calpe, 1936. 274 pp. BH, xxxrx, 171172.

467. Barbitt, Theodore. La crónica de Veinte Reyes. A comparison with the text of the Primera Cronica general and a study of the principal latin sources. New Haven, Yale University Press, 1936. x-172 pp. $\mathrm{BH}, \mathrm{XxxIx}, 258-261$. 
468. Crews, Cynthia M. Recbercbes sur le judéo-espagnol dans les pays balkaniques. Paris, E. Droz, 1935. 321 pp. BH, xxxIx, 268-269.

469. Cuvelier, J. et J. Lefèvre. Correspondence de la Cour d'Espagne sur les affaires des Pays-Bas au XVIIe siècle. Recueil commencé par Henri Lonechay et continue par Joseph Cuvelier avec la collaboration de Joseph Lefèvre. Tome V: Précis de la correspondence de Charles II (1665-1700). Bruxelles, Maurice Lamertin, 1935. xxv799 pp. BH, xxxIx, 177-179.

470. Dudon, P. Paul. Saint Ignace de Loyola, par... Deuxième édition. Paris, Gabriel Beauchesne et fils, 1937. xx-663 pp. BH, xxxrx, 172-175.

471. Espinosa, hijo, Aurelio M. Arcaismos dialectales. La conservación de "s" $y$ " $z$ " sonoras en Cáceres y Salamanca por . . bijo. Madrid, Hernando, 1935 . xxxii-256 pp. BH, $\mathrm{xxxIx}, 77-79$.

472. Farinelli, A. Lope de Vega en Alemania. Traducción de la obra alemana "Grillparzer und Lope de Vega", por Enrique Massaguer, con una carta del autor al traductor y un artículo de Marcelino Menéndez y Pelayo. Barcelona, Bosch, 1936. 325 pp. BH, xxxix, 176-177.

473. García Rey, Verardo. Archivo de Tradiciones populares. IV: Vocabulario del Bierzo. Madrid, Centro de Estudios Históricos, 1934. 163 pp. BH, xxxrx, 75-77.

474. Giese, W. Nordost-Cadiz, ein Kulturwissenshaftlicher Beitrag zur Erforscbung Andalusiens. Halle, Max Niemeyer Verlag, 1937. viii254 pp. BH, $\mathrm{xxxIx}, 411-412$.

475. González-Llubera, Ignacio. Coplas de Yoçef, a medieval-Spanish poem in bebrew characters, edited with an introduction and notes by... Cambridge, At the University Press, 1935 . xxi-50 pp. BH, XxxIx, 261-265. (Con Henry V. Besso.)

476. Guillaumie-Reicher, Gilberte. Le voyage de Victor Hugo en 1843. France-Espagne-Pays basque. Paris, E. Droz, 1936. iv-252 pp. BH, XXXIX, 282. 
477. Henriquez Ureña, P. La cultura y las letras coloniales en Santo Domingo. Buenos Aires, Biblioteca de Dialectología hispanoamericana, Anejo II, 1936. 193 pp. BH, xxxIx, 414-415.

478. Horsman, Gertruida Christine. Luis Zapata. Varia bistoria (miscelánea). I. (Proefschrift ter verkrijgin van den graad van Doctor in de Letteren.) Amsterdam, H. J. W. Becht, 1935. xxxix-175 pp. $\mathrm{BH}, \mathrm{xxxIx}, 271-272$.

479. Jörden, Dr. Otto. Die formen des Sonetts bei Lope de Vega, von... Halle, Max Niemeyer Verlag, 1936. xii-372 pp. BH, xxxıx, 276277.

480. Krüger, Fritz. Die Hochpyrenäien, C. Landliche Arbeit. Band I: Transport und Trangsportgeräte . . Hamburg, Conrad Behre, 1936. 202 pp. BH, $\mathrm{xxxxx}, 283-284$.

481. Levi, Ezio. Lope de Vega e l'Italia, con prefazione di Luigi Pirandello. Florencia, G. C. Sansoni, 1935. xiv-viii-174 pp. BH, xxxrx, $175-176$.

482. López de Vega, Antonio. Paradoxas racionales escritas en forma de diálogos, del género narrativo la primera, del activo las demás, entre un cortesano y un filósofo, editadas con una introducción por Erasmo Buceta. Madrid, Hernando (1937). xliii-138 pp. BH, xxxIx, $68-70$.

483. McClelland, I. L. The origins of the romantic movement in Spain. Liverpool, Institute of Hispanic Studies, 1937. xii-402 pp. BH, xxxIx, 418-420.

484. Martínez y Martínez, Francisco. Don Guillén de Castro no pudo ser Alonso Fernández de Avellaneda. Réplica al folleto de D. Emilio Cotarelo y Mori "Sobre el Quijote de Avellaneda y acerca de su autor verdadero." Valencia, 1935. 119 pp. BH, xxxıx, 277-279.

485. Mérimée, Paul. L' influence française en Espagne au XVIIIe siècle. Paris, Societé d'édition "Les Belles-Lettres", (1937). 128 pp. BH, XxxIX, 280 .

486. Mesnard, Pierre. L'essor de la pbilosopbie politique au XVIe siècle. Paris, Boivin, 1936. viii-711 pp. BH, xxxIx, 272-273. 
487. Mez, A. El Renacimiento del Islam. Traducción del alemán por Salvador Vila. Madrid, Imprenta Maestre, 1936. viii-463 pp. BH, XxxIx, 256-258.

488. Pagès, A. La poésie française en Catalogne du XVIIe. siècle à la fin du XVe. Etudes suivies de textes inédits ou publiés d'après les manuscrits... par... Toulouse, E. Privat. n. d. n. p. BH, xxxIx, 412-414.

489. Prestage, editor Edgar. Chapters in Anglo-Portuguese relations. London, Warford, Voss and Michael Ltd., 1935. vii-198 pp. BH, XXXIX, 279-280.

490. Reparaz Ruiz, Gonzalo de. España. La tierra, el bombre, el arte. Tomo I. Barcelona, Editorial Martín, 1937. 168 pp. BH, xxxrx, $285-286$

491. Roberts, Graves Baxter. The epithet in Spanish poetry of the romantic period. University of Iowa Studies, No. 6., 1936. 166 pp. BH, xxxIx, 417-418.

492. San Román, Francisco de B. El archivo bistórico provincial de Toledo. I: Los protocolos de los aníiguos escribanos de la Ciudad imperial. Notas e indices por... II. Lope de Vega, los cómicos toledanos y el poeta sastre. Los publica y comenta... Madrid, Imprenta Góngora, 1934. 174 y 256 pp. BH, xxxIx, 273-275.

493. Serra-Vilaró, J. Fructuós, Auguri i Eulogi Mártirs Sants de Tarragóna. Taragona, Tallers Torres i Virgili, 1936. 294 pp. BH, xxxix, $255-256$.

494. Serrano, D. Luciano. El obispado de Burgos y Castilla primitiva desde el siglo $V$ al XIII. Tomos I y II. Madrid, 1935; Tomo III, 1936. 488,452 y 480 pp. BH, xxxix, 164-167.

495. Shoemaker, W. H. The multiple stage in Spain during the fifteenth and sixteenth centuries. Princeton, University Press, 1935. xi-150 pp. BH, $\mathrm{xxxIx}, 179-180$.

496. Torre, Martín de la y Pedro Longas. Catálogo de Códices latinos. Tomo I: Biblicos. Madrid, Patronato de la Biblioteca Nacional, 1935. xvi-417 pp. BH, xxxIx, 167-171. 
1938

497. La Chronique générale et le poème du Cid. BH, xL, 306-309.

498. L'epître de Bartolomé Leonardo de Argensola "Dicesme Nuño." $\mathrm{BH}, \mathrm{xI}, 48-61$.

499. La mauropbilie littéraire en Espagne au XVIe siècle. $\mathrm{BH}, \mathrm{xL}, 150-$ $158 ; 281-296 ; 433-448$.

500. Rapport sur l'activité de l'Ecole des Hautes Etudes Hispaniques de Madrid par... Doyen. Année scolaire 1936-1937. Bordeaux, Imprimerie Delmas, 1938. $16 \mathrm{pp}$.

$R$ e $s$ e กี a $s$ :

501. Voigt, P. Die Sierra Nevada. Hans, Hansrat, Hansliches und gewerbliches Tagewerk... Hamburg, Paul Evert Verlag, 1937. BH, xL, 106.

\section{9}

502. Les "Anales de la Corona de Aragón" de Jerónimo Zurita. $\mathrm{BH}, \mathrm{XII}$, 126-14.1.

503. Les fils D'Arias González (Episode final du siège de Zamora), d'aṕès la Chronique générale et Gil de Zamora. BH, XuI, 266-269.

504. La mauropbilie littéraire en Espagne au XVIe siècle. $\mathrm{BH}, \mathrm{xLI}, 65-$ $85 ; 345-351$.

505. Pierre Paris et la Casa Velásquez. BH, xLI, 368-370.

506. Quelques mots encore sur le Cid. BH, XLI, 178-180.

507. Le vrai Cid. BH, xLr, 86-89.

Reseñ a :

508. Bertini, G. M. Tirso de Molina, El Condenado por desconfiado, a cura di G. M. Bertini. Torini, G. B. Paravia \& Co. (Universita di Torino. Publicazioni della Faculta di Magisterio.) xli-192 pp. BH, $\mathrm{XLI}, 193-196$. 
509. Kany, Ch. E. The beginnings of the Epistolary novel in France, Italy and Spain. Berkeley, Calif., University of California Press, 1937. x-158 pp. BH, xLI, 191-193.

510. Lagrone, Gregory Gouch. The imitations of "Don Quixote" in the Spanish drama. Philadelphia, University of Penssylvania Press, 1937. vii-145 pp. BH, xLI, 380.

511. Lynn, Caro. A College professor of the Renaissance, Lucio Marineo Siculo among the Spanish Humanists. Chicago, The University of Chicago Press, 1937. ix-302 pp. BH, xLI, 90-95.

\section{0}

512. L'Auto de la Pasión de Lucas Fernández. BH, XLII, no. 4, 285-291.

513. La maurophilie littéraire en Espagne au XVIe siècle. BH, XLII, 213227.

514. Quelques mots encore sur les "Maris jaloux" de Cervantes. BH, XIII, 303-306.

515. Quelques publications récentes sur les "Sephardim." BH, XLII, 140152.

516. Rapports sur l'activité de l'Ecole des Hautes Etudes Hispaniques au cours des années 1936-1940. BH, XLII, 307-327.

517. La veine épique en Portugal et en Espagne. BH, XIII, 136-139.

R e s e $\tilde{n}$ a $s:$

518. Abraham, Richard. A portuguese version of Barlaam and Josapbat. Paleographical edition and linguistic study. Philadelphia, University of Pennsylvania Press, 1938. xv-144 pp. BH, xLII, 62-64.

519. Altamira, Rafael. La civilización española en los siglos XVI, XVII y XVIII. Tirada aparte de los tomos segundo y tercero de la Historia de la Nación Argentina. Buenos Aires, Imprenta de la Universidad, 1937. 100 pp. BH, XIII, 182.

520. Brasillach, R. et Maurice Bardèche. Histoire de la guerre d'Espagne. Paris, Plon, 1939. vi-442 pp. BH, XIII, 184-186. 
521. Bushee, Alice Huntington. Three centuries of Tirso de Molina. Philadelphia, University of Pennsylvania Press, 1939. x-111 pp. $\mathrm{BH}$, xurr, 171-173.

522. Casalduero, Joaquín. Contribución al estudio del tema de Don Juan en el teatro español. Northampton, Mass., Smith College, 1938. viii-108 pp. BH, XIII, 173-175.

523. Cortés, N. Alonso. Sumandos bibliográficos. Gómez Manrique, Berruguete. Fray Bartolomé de las Casas. Hernando de Cangas. El Padre Isla. García de la Huerta. López de Sedano. Larra. Valladolid, Librería Santaren, 1939. 146 pp. BH, XLII, 168-169.

524. Coutinho, Bernardo Xavier' C. Bibliographie franco-portugaise. Essai d'une Bibliograpbie chronologique de livres français sur le Portugal. Porto, Librairie Lopes da Silva, 1939. xiii-412 pp. BH, XLII, 66-67.

525. Doutreport, Georges. Chroniques de Jean Molinet publiées par... T. III. Introduction, glossaire, Index. Bruxelles, Palais des Académies, 1937. viii-451 pp.

526. Hanke, Lewis. Handbook of Latin American Studies. 1936. Edited by... Cambridge, Harvard University Press, 1937. xv-517 pp. BH, XLII, 262-263.

527. Keniston, Hayward. The Syntax of Castilian prose. The sixteenth century. Chicago, The University of Chicago Press, 1937. xxix750 pp. BH, XLII, 162-165.

528. Lecoy, Félix. Recbercbes sur le "Libro de Buen Amor" de Juan Ruiz archiprêtre de Hita. Paris, Librairie E. Droz, 1938. 374 pp. BH, XLII, 155-162.

529. Levi-Provençal, E. La civilisation arabe en Espagne. Vue générale. Le Caire, 1938. 208 pp. BH, xum, 153-155.

530. Luquiens, Frederic Bliss. Spanish American literature in the Yale University Library. A bibliograpby. New Haven, Yale University Press, 1939. x-335 pp. BH, xLII, 183-184.

531. Lynch, Charles H. Saint Braulio, bishop of Saragossa (631-651), bis life and writings. Washington, D. C., The Catholic University of America, 1938. xii-276 pp. BH, xill, 241-247. 
532. Menéndez Pidal, Ramón. La España del Cid. Madrid, Espasa-Calpe, s. a., xv-505 pp. BH, XLII, 248-249.

533. Read, J. Lloyd. The Mexican bistorical novel, 1826-1910. New York, Instituto de las Españas en los Estados Unidos, 1939. xiv337 pp. BH, XLII, 341-342.

534. Restrepo, P. Félix. El alma de las palabras. Diseño de semántica general, por el... Segunda edición. Bogotá, Librería Voluntad, 1939. 244 pp. BH, XLII, 170-171.

535. S. Amour, Sister Mary Paulina. A study of the Villoncico up to Lope de Vega: Its evolution from profane to sacred thenes, and specifically to the Christmas Carol. Washington, D. C., The Catholic University of America Press, 1940. X-131 pp. BI, XIIr, 253258.

536. Silvestre, Gregorio. Gregorio Silvestre, estudio biografico y critico, por Antonio Marin y Ocete. Granada, Publicaciones de la Facultad de Letras, 1939. 272 pp. BH, XLM, 176-178.

537. Spell, Jefferson Rea. Rousseau in the Spanish world before 1833. A study in Franco-Spanish literary relations. Austin, University of Texas Press, 1938. 325 pp. BH, xirn, 180-182.

538. Stevens, Ch. H. Lope de Vega's El Palacio confuso, together with a study of the Menaechmi theme in Spanish literature. New York, Instituto de las Españas en los Estados Unidos, 1939. xcii-138 pp. BH, XII, 178-180.

539. Zaldumbide, Gonzalo. Montalvo y Rodó. New York, Instituto de las Españas en los Estados Unidos, 1938.283 pp. BH, XLI, 263265.

540. Zellers, Guillermo. La novela bistónica en España, 1828-1850. New York, Instituto de las Españas en los Estados Unidos, 1938. 168 pp. BH, xKII, 182-183.

\section{1}

541. A Propos d'Encina. Coup d'oeil sur notre vieux drame religieux. $\mathrm{BH}, \mathrm{xLIII}, 123-151$. 
542. "El gran teatro del mundo." BH, xLIII, 290-305.

543. La "loa" de "La vida es sueño." BH, XIII, 65-71.

544. La maurophilie littéraire en Espagne au XVIe siècle. BH, XLIm, 265 289.

545. Le théâtre religieux d'Encina. BH, xuII, 5-35.

546. Zanfoña et Zampoña. BH, XIm, 152-161.

Res e $\tilde{\mathbf{n}}$ a $s$ :

547. Saavedra Molina, Julio y Erwin K. Mapes, editors. Obras escogidas de Rubén Dario publicadas en Cbile. Tomo I. Abrojos, Canto épico, Rimas, Azul. Santiago de Chile, Universidad de Chile, 1939. 408 pp. BH, XriII, 317-318.

548. Salinas, Pedro. Reality and the Poet in Spanish poetry. Baltimore, The Johns Hopkins Press, 1940. 165 pp. BH, XLII, 314-317.

\section{2}

549. L'allégorie des tireurs à l'arc. BH, $\mathrm{xLIV}, 171-174$.

550. L'bumeur de Berceo. BH, XIIV, 160-165.

551. La mauropbilie littéraire en Espagne au XVIe siècle. BH, $\mathrm{xLIV}, 96-$ 102.

552. Rapport sur le fonctionnement de l'Ecole des Hautes Etudes Hispaniques pour l'année 1939-1940. BH, xurv, 181-184.

553. Ronsard et les espagnols. BH, XLIV, 168-170.

554. Sur le "Mester de Clerecia." BH, xurv, 5-16.

555. Le style de Vélez de Guevara. $\mathrm{BH}, \mathrm{x} \perp \mathrm{xv}, 175-180$.

556. La citola. BH, XLIV, 77-80.

1943

557. L'épisode de Doña Endrina dans le "Libro de Buen Amor." BH, $\mathrm{XLV}, 139-156$. 
558. Pour combler les lacunes de l'bistoire du drame religieux en Espagne avant Gómez Manrique. $\mathrm{BH}, \mathrm{XLv}, 55-62$.

559. Le procedé dans "El Diablo cojuelo." BH, xLV, 69-72.

560. Rapport sur le fonctionnement de l'Ecole des Hautes Etudes Hispaniques pour l'année 1941-1942. BH, xLv, 202-204.

\section{4}

561. A propos du "Diablo cojuelo." Aperçus de stylistique comparée. $\mathrm{BH}, \mathrm{XLVI}, 240-251$.

562. Une bistoire de captifs dans le "Diable boiteux" de Lesage. BH, XIVI, 26-34.

563. La maurophilie littéraire en Espagne au XVIe siècle. $\mathrm{BH}, \mathrm{xLV}, 5-$ 25.

564. Rapport sur l'activité de l'Ecole des Hautes Etudes Hispaniques pour l'année 1942-1943. BH, xLvi, 73-78.

565. Rapport sur l'activité de l'Ecole des Hautes Etudes Hispaniques pour l'année 1943-1944. BH, xLvr, 252-254.

R e s e ñ a $s$ :

566. Estelrich, J. Vivès. Exposition organisée à la Bibliothèque Nationale, Paris, janvier-mars, 1941. Dijon, Imprimerie Darantière, 1942. xix194-26 pp. BH, XLVI, 93-95.

567. Montesinos, J. F. et Ch. V. Aubrun. Lope de Vega. Peribañez y el Comendador de Ocaña, tragi-comedia. Texte original publié avec une notice, une étude littéraire et des notes par Ch. V. Aubrun et J. F. Montesinos. Paris, Librairie Hachette, (1944). BH, xLVI, 274276.

568. Menéndez Pidal, Ramón. La lengua de Cristóbal Colón, el estilo de Santa Teresa y otros estudios sobre el siglo XVI. (Madrid), Espasa-Calpe, 1942. 176 pp. BH, xIVI, 260-262.

569. Pagès, editor Amedée. Cbronique catalane de Pierre IV d'Aragón III de Catalogne, dit le Cérémonieux ou del Punyalet. Bibliothèque méridionale, publiée sous les auspices de la Faculté des Lettres de 
Toulouse. 2ème. serie, tome XXXI. Toulouse, E. Privat, 1942. 1xxii472 pp. BH, XLvi, 87-92.

570. Sánchez Alonso, B. Historia de la bistoriografia-española. I. Hasta la publicación de la Crónica de Ocampo. Madrid, 1941. 478 pp. (Publicaciones de la "Revista de Filologia Española".) BH, XIVr, 79-84.

571. Terlingen, Johannes Hermanus. Los italianismos en español desde la formación del idioma hasta principios del siglo XVII. Amsterdam, N. V. Noord-Hollandsche Uitgeversmaatschappij, 1943, xii400 pp. BH, xIVr, 101-103.

572. Yo en Cate. Poema de Alfonso XI publicado por... doctora en Letras y Filosofía. Tomo I. Estudio preliminar y vocabulario. Amsterdam, N. V. Swets \& Zeitlinger, 1942. xxiii-194 pp. BH, XLVr, 84-87.

\section{5}

573. "Cantares" et "Romances" (simple aperçu). BH, XIVII, 1-25; no. 2, 169-186.

574. L'Episode des Infants de Carrión (L'Affaire du Lion et la scène des adieux), dans le "Mio Cid" et la Chronique générale. $\mathrm{BH}, \mathrm{xLvII}$ 124-133.

R e s e ñ a $s$ :

575. Molho, Michael. Le Méam-Loez, encyclopédie populaire du sepharadisme levantin. Thessalonique 11945. 28 pp. BH, xLvir, 238.

\section{6}

576. L'affaire des malles du Cid. BH, xLvu, 170-177.

577. Coup d'oeil sur la poésie épique du siècle d'or. BH, xцvır, 294-329.

578. L'Episode des Infants de Carrión (La rouvraie de corpes et le retour a Valence) dans le "Mio Cid" et la Chronique générale. $\mathrm{BH}, \mathrm{xL} v \mathrm{II}$, 64-74.

579. Inventaire estimatif du "Mester de Clerecia." BH, XLVII, 193-209. 


\section{NOTAS}

1. Cf. L. Barrau-Dihigo, en: Revue Hispanique, 1906, XIII, no, 43, p. 261 .

2. Cf. A. Ludwig, en: Archiv für das Studium det Neveren Sprachen und Literaturen, 1906, LX, Jahrgang, 220-224.

3. Publicado separadamente por Féret \& Fils, Bordeaux, s. d.; 20 pp.

4. El artículo final, conclusión de ese estudio, debió de aparecer en la Revue Historique de Bordeaux, después de 1920; pero no se halló en la Biblioteca del Congreso, para registrarlo. Se sabe, sin embargo, que el estudio completo apareció después en forma de libro, publicado por Féret 8 Fils, de Bordeaux. 


\section{N D I C E E N ER A L.}

Los números que van después de cada nombre se refieren a los que se hallan antes de cada título en la Bibliografía. Los títulos en cursiva son de trabajos originales de M. Georges Cirot; los de tipo común, son de reseñas críticas. Los nombres de autores ctyos trabajos fueron reseñados por M. Cirot están en versalitas; los nombres de lugares, instituciones, títulos y periódicos, en itálicas.

\section{A}

Aben Fumeya, 432.

Abencertaje, Historia del, 325.

ABRAHAM, Richard, 518.

ABUBEQUER de Tortosa, 364.

ACEVEDO Y HUELVES, Bernardo, 398.

ADAMS, N. B., 228.

Africa y Amética, 196.

Ajedrez, Libro de, 458.

AL SAOUNDI, 445.

Alabanza de aldea, 152.

ALARCón, Maximiliano, 364.

ALBA, el Duque de, 210, 234, 222.

ALEMAN, Mateo, 91.

ALFARABI, 399.

ALFONSO $X$, el sabio, 205.

ALFONSO XI, Poema de, 572.

"Aljamiado" et "ladino", 442.

ALPERN, Hymen, 273.

ALONSO, Amado, 435, 446, 462.

Alphonse VIII, Anecdotes ou légendes sur . . 223, 252, 268.

ALTAMIRA, Rafael, 178, 198, 400 , 519.

RMADÉ, Jean, 229.

América latina, El porventr de la, 60 .

Americanismos, 161.

AMEZÚA, A. de., $380,447$.

Analecta Montserratensia, 144.

Anales de la Corona de Aragón, 502.

ANDERSSON, Thomas; 448.
ANDRENIO, 145.

ANGLÈS, H., 245.

ANABAL, C. E., 255.

ANSPACH, Eduard, 381.

ANTOLfN, G., 67.

Anuario de Historia del Derecho espa. nol, $271,289$.

Arabe à Botdeaux, L'enseignement de, 135.

Aragón, Almitantes de 171.

Aragón, Documentos para la historia de, 92 .

ARANHA, Brito, 15.

Atcaismos dialectales, 471.

ARGENSOLA, Bartolomé Leonardo, 498.

ARGENSOLA, Lupercio Leonardo de, 319.

Atpa de David, El, 255.

Arthutian legend in Spanish literature, 276.

ARTIGAS, Miguel, 230, 335.

Asín, J. Oliver, 309.

Aś́N PALACIOS, Miguel; 302;401, 423.

ATKINSON, W. C., 402 .

AUBRUN, Ch. V., $450,560$.

AUGÉ, Claude, 103.

AURÈS, Paul, 153.

Auto de la Pasión, 512. 
B

BABELON, J., 87.

BACH Y RITA, Pedro, 365.

BAER, Fritz, 310.

BAILLY, Ch., 465.

BALBUENA, Bernardo de, 320, 387.

BALSEIRO, José A., 424.

Ballads, Spanish, 70.

BALLESTER Y CASTELL, R. 290.

BALLESTEROS, M. G. de, 291, 466.

BANES, M. Fr. Domingo, 199.

BARBITT, Theodoro, 467.

BARDÈCHE, Maurice, 520.

BARJA, César, 231, 256.

Batlaam and Josaphat, 349, 456, 518.

BARRAU-DIHIGO, J., 26.

BATTISTESSA, A. J., 313.

BAYER Y SALAMANCA, Pérez, 167, 1. c.

BELTRÁN Y RÓZPIDE, Ricardo, 184. BELL, Aubrey F. G., 200, 232, 233 . 257.

BELLO, Andrés, 6.

BËNOLIEL, Joseph, 417.

Berceo, Cuatro poemas de, 297; El humour de, 550; Veintrés milagtos, 336.

BERNARD, Augustín, 76.

Berruguete y su obra, 157.

BERTINI, G. M., 407, 508.

BERTRAND, L., 403.

BERWICK Y DE ALBA, Duque de, 146 , $210,234,272,422$.

BESsO, Henry V., 475.

Biblia castellana de... Guadatajara 234.

Biblia romanceada, 313.

Bibliographie franco-portugaise, 524.

Biblioteca del Escorial . . catálogos latinos de la, 67; códices de la, 140.

Biblioteca nacional, Manuscritos de América en la, 436.

BLÁZQUEZ Y NGUILERA, Antonio, 186.

BOHIGAS-BALAGUER, R., 235.

BOISSONNADE, E., 185 .

Biblioteca Menéndez y Pelayo, Boletín de la, 147.

Bolivar, Lettres et discours. . , 450.

BONA, R., 77.

BONILLA Y SAN MARTIN, Bonilla, 250 , 305.

Bonilla y San Martín, Homenaje a, 305.

BOUILLIER, V., 239.

BRASILLACH, R., 520 .
Brasil restituido, El, 358 .

BRATLI, Ch., 78.

Brunot, Ferdinand. Mélanges de philologie offerts à ..., 22.

Brutails, J. A., 223 a.

BUCETA, Erasmo, 482.

BULLON Y FERNÁNDEZ, Eloy, 292. 337.

Burlas veras, Las, 100.

BURNAM, J. M., 88, 236.

BUSHEE, Alice Huntington, 521.

\section{C}

Calatrava, Romances del Maestre de, 363.

CALCOTT, Frank, 205.

Calila y Dimna, 175.

Cancioneto de lírica asturiana, 203.

Cancionero del Seih..., 413.

CANO, Juan, 311.

Cantar de gesta, 294.

"Cantares" et "Romances", 573.

CAPELLANI, Andreae, 366 .

Catpio, Bernardo del, 294.

CARVAJAL, Micael de, 404.

CARRERAS Y CANDI, F., 89.

CARRIAZO, Juan de, 282.

CASALDUERO, Joaquín, 522.

Castigo sin venganza, El, 154.

Castellana, Sintaxis de la, 527.

CASTRO, Adolfo de, 7.

CASTRO, Américo, 148, 186, 207. $258,312,313,367$.

CASTRO, Guillén de, 273, 484 .

CASTRO GUISASOLA, F., 237.

Castigo sin venganza, El, 314.

Catálogo de la Biblioteca Nacional, 25.

Catálogo de la Real Biblioteca, 10.

Catalunya... geografia general, 89.

Cauallero Zifar, Libro del, 360.

Cavalleto de Dios, Libro del, 360.

Cedillo, Conde de, 11.

CEJADOR Y FRAUCA, Julio, 79.

Celestine, La, 192: Fuentes literarias de la, 237.

Celoso extremeño, El, 284.

CONOVER, Helen, II.

CERNY, Václav, 459.

CERVANTES, Miguel de, 93, 142, 159. $286,287,258,348,367,386$, 439,514 .

CETINA, Gutierre de, 251.

Charles Quint, Historiogtaphie de, 80.

Chanson de Roland, La, 185.

CHATILLON, Gautier de, 462. 
Chronique latine des rois de Castille, $61,110,114,122,126,180$.

Chronique léonaise inédite, La, 53, 82, $105,106,123,406$

Cid, Biographie du, 81; Chronique générale et le, 497; Courtly Hero, 298; Poème du, 191; Cf. también, 507, 574, 576, 578.

Ciencia genealógica, Origen de la, 339. Ciprián de Sanabria, Dialecto de San, 243.

CIROT, Georges Eugène Alfred, 1, 2 , $3,4,5,12,13,18,20,21,22$, $23,24,27,28,29,30,31,32$, $37,38,39,40,41,42,43,46$, $47,48,53,54,55,61,62,63$, $64,65,66,71,72,73,74,75$, $81,82,83,84,85,86,105,106$, $107,108,109,110,111,112$, $113,114,115,120,121,122$, $123,124,125,126,127,133$, $134,135,136,137,138,142$. $143,162,163,164,165,175 \mathrm{a}$, $176,177,178,179,180,181$, $182,183,197,198,199,204$, 223,223 a, 224, 225, 226, 227, $252,254,268,269,270,283$, $284,285,286,287,288,305$, $306,307,308,330,331,332$, $333,334,361,362,363,378$. $379,396,397,417,418,419$, $420,421,439,440,441,442$, $443,444,463,497,498,499$, $500,502,503,504,505,506$, $507,512,513,514,515,516$, $517,541,542,543,544,545$, $546,549,550,551,552,553$, $554,555,556,557,558,559$, $560,561,562,563,564,565$, $573,574,576,577,578,579$.

Citola, La, 556.

Civilisation arabe en Espagne, La, 529. Clásicos castellanos, 206, 207.

Códices latinos, Catálogo de, 496.

Colomb, Fetnand ... Bibliothèque de, 87.

Colón, La lengua de Cristóbal, 568.

Colleçấo de manuscriptos . . , 68 .

Comedia Ypolita, The, 315.

Comic types in Spanish drama, 213.

Conde Lucanor, El, 174.

Condenado por desconfiado, El, 508.

Conjuración de Venecia, La, 432.

Conquista americana, Mitos de la, 340.

Coplas de Yoçef, 475.

CORBIÉRE, A. S., 274.

Cordobés valeroso, El, 322.
Corona merecida, La, 222.

CORTÉs, N. Alonso, 207, 208, 259. $425,523$.

cossío, J. M. de, 382 .

COSTE, René, 113.

COTARELO Y MORI, Emilio, 484.

COULET, J., 49.

COUR, Aug., 166.

COUTINHO, Bernardo Xavier C., 524.

CRAWFORD, J. P. Wichersham, 209.

CREWs, Cynthia M., 468.

CROCE, San Giovanni della, 405.

Crónica general de España, 56.

Cuba, Historia de, 201.

Cuerdo loco, El, 222.

CUERVo, Rufino José, 6.

CUVELIER, Joseph, 215, 293, 369, 406,469 .

DAM, C. F. Adolfo Van, 314.

Dama boba, La, 154.

Dario Rubén, Influence française dans, 279, 452; Obras escogidas de, 547.

DAUMET, G., 90.

DAVIDS, J. A., 368 .

De proprietate sermonum, 9.

DEFERRARI, Harry Austin, 275.

DEMIMUID, Mgr., 149.

Desdén vengado, El, 333.

Diccionario hispánico etimológico, 238.

Diego de Castilla, Deán don, 316.

DOMfNGUEZ BERRUETA, J., 150.

DOUGLASS, Philip Earle, 305.

DOUTREPORT, Georges, 525.

DUDON, P. Paul, 470.

DUFOURCQ, A., 1.

DURÁN Y SAMPERE, A. 383.

\section{E}

Ecole des Hautes Etudes Hispaniques de Madrid, Rapport sur l', 500 , $516,552,560$.

Encina et le drame religieux, 541, 545 .

Enrique IV de Castilla, 335.

Entremés de los Romances, 338.

Entremés in Spain, Early, 221.

ENTWISTLE, William J., 276, 294.

Epistolar, novela en Francia . . . 509:

España, Historia de, 17, 453, 490: Espagne et les Pays-Bas, 215, 293, 369; España y Portugal, 338; As duas Espanhas, 384: España del Cid. 299, 336, 532: Espagne musulma. ne, 412 . 
Español, Orígenes del, 321.

FSPINOSA, hijo, Aurelio M., 471.

Espionnage en Espagne, 111.

ESTELRICH, J., 566.

Estrella de Sevilla, La, 154, 390.

Estudios Universitarios Catalans, 175 a.

Eugenie, Lettres de l'Impétatrice, 422.

EYZAGUIRRE ROUSE, G., 56.

EZGUERRA DEL BAYO, J., 210.

\section{F}

FARINELLI, Arturo, 108, 187, 295, $338,407,472$.

Feijóo, Ideas biológicas del Padre, 431.

Fernán González, 270, 306, 334:

FERNÁNDEZ, Lucas,.. 512.

FERNÁNDEZ DE AVELLANEDA, Aionso, 484 .

FERNANDEZ DE HEREDIA, Juan, 319.

FERNÁNDEZ DE VEL ÂSCO, D. Pedro, 285.

FERNÁNDEZ Y FERNÁNDEZ, Marcelino, 398.

FÉROTIN, Dom Marius, 16.

FICHTER, William, 260.

FIGUEIREDO, F, de, 104, 384.

FITZMAURICE-KELLY, J., 211, 212.

Floite et Blanceflor, L'Histoire de, 284.

Flores, Novelas de Juan de, 371 .

FORD. J. D. M., 385, 386, 426.

FORT, Ramón, 2.

FRANKOWSKI, Eugeniusz, 151.

Fuero de Madtid, 408.

Fueros de Castilla, Libro de los, 248.

Fueros de Soria y Alcalá de Henares, 160.

FUETER, E., 69.

\section{G}

GALIARDO, Bartolomé José, 316.

GANDÍA, E. de, 339, 340 .

GARCIA, E. Juan, 167.

GARCÍA DE DIEGO, Vicente, 242, 238.

GARCÍA GÓMEZ, Emilio, 307, 445.

Garcia Gutiértez, Dtamas de, 228.

GARCIA REY, Verardo, 473.

Gatcilaso de la Vega, Publications sut, 133.

GIESE, W., 474 .

GILLET, Joseph E., 404.

GIULIAN, A. A., 341 .

Gloria d'amot; La, 139.

Gómez de Avellaneda, Gertrudis, 267.

Gómez Ocerín, J., 222.

Góngora, Luis de, 230, 338, 322. $446,464,376$.
González, Vida de Estebanillo, 434.

GONZÁLEZ-LLUBERA, Ignacio, 253 , $388,475$.

GONZÁleZ Palencia, A., 188, 399 , $308,332,419$.

GRACIÁN, Baltasar, 239, 200.

Gtamática castellana, 6 .

Gramática de la lengua castellana, 253.

Granada, Fr. Luis de, 294.

GREEN, Otis Howard, 309.

Gtillpatzer und Lope de Vega, 472.

GROSSMANN, R., 277.

GROULT, P., 296.

Guatani, Hispanismos en el, 435 .

GUERRA, J. C., 410.

Guetta de Gtanada, 136.

GUERRA Y SÁNCHEZ, R., 201.

Guevara, Le style de Vélez de, 555.

GUILLAUMIE-REICHER, Gilberte, 476.

GUINARD, Paul, 427.

Guzmán de Alfarache, 91.

\section{$\mathrm{H}$}

HAINSWORTH, G., 428.

HAMEL, Adalbert, 212.

Handbook of Latin American Studies, 526.

HANKE, Lewis, 526.

HANOTAUX, Gabriel, 422.

HARDY, G., 153.

HARLAN, Marcel Margaret, 343.

Hartzenbusch, Juan Eugenio, 274:

HAUSER, Henti, 411.

Hayton, Prince of Gorigos, 451.

HEATON, H. C., 139 .

HENDRIX, W. Samuel, 213.

HENRÍQUEZ UREÑA, P., 477.

HERMOSILLA, Diego de, 168.

Hésperis, Archives Berbères, 169.

Hispania (Paris), 138.

Hispanic-Ametican History, List of works, 189.

Hispanic Society of America, 211.

Historia da colonização do Brazil, 240.

Histotia de España, Manual de, 400 . 403.

Historia de la nación argentina, 519.

Historiographie espagnole, $L^{\prime}, 20,23$.

Historiographie hispano-portugaise, $419,444$.

Hita, Atcipreste de, 79, 528.

HOL.LE, Fritz, 91.

HOORNAERT, R., 214.

HORNE, J. Van, $320,387$.

HORSMAN, Gertruida Christine, 478. 
Hugo, Voyage de Victor... en 1843 , 476.

Humor in story \& essay, Spanish, 173. HURTADO, J., 188.

\section{I}

IBARRA, Eduardo, 278.

IBARRA RODRÍGUEZ, E., 92, 170

Ibn Hazan al-Andalusi, Abu Muhammad Ali, 3.72.

IBN SABARA, Joseph Benmeir, 388.

Ignace de Loyola, San, 2, 470.

Infantes de Lata, La leyenda de los, 433.

Institut d'Etudes Hispaniques de $\mathrm{Pa}$ ris, Inaugutation de $l^{\prime}, 288$.

Institut français de Madrid, 72.

IRISARRY HONORAT, B., 8.

ISAZA Y CALDERON, B., 429.

Islam español, El, 445.

Italia e Spagna, 295.

Italianismos en el español, Los, 571.

\section{J}

John III of Pottugal, Letters of, 385 , 426.

JÖRDER, Otto, 479.

Juan, Estudio del tema de Don, 522.

Juan de la Cruz, San, 375.

Juan Manuel, Don, 174.

Jüden in Christlichen Spanien, 310.

Judéo-espagnol dans les Pays Balkaniques, 468 .

Judeo-español de Otiente, Caracteres generales del, 395.

Judeo-spanish, Monastit dialect of, 395.

Juifs espagnols et pottugais à Bordeaux, $29,31,39,42,165,297,417$.

Juive de Tolède, La, 162.

\section{K}

KANY, Ch. E., 509.

KENISTON, Hayward, $189,527$.

KING, Georgiana G., 241.

KREPINSKY, M., 242.

KRÜGER, Fritz, 243, 480.

\section{L}

LABRA, R. M. de, 57.

"Ladino" et "aljamiado", 442.

LAGRONE, Gregory Gouch, 510.
LAMBERT, Elie, 389.

Lámpara de los Principes, 364.

Langue espagnole en Argentine, 415.

LANSING, Ruth, 386.

LANZ, L. Valenilla, 450.

Laumonier, Paul (Mélanges), 421.

LAURENCín, Marqués de, 171.

Lazarillo de Tormes, Vida del, 102.

LE GENTIL, Georges, 430.

LEAVITT, Sturgis E., 390.

LECOY, Felix, 528.

LEFÈVRE, J., 293, 359, 406.

LEITE DE VASCONCELLOS, J., 3, 50.

Lengua castellana, Apologías de la, 341.

Lengua francesa. Conjugación de la, 8.

León, Luis de, 257.

LEVI, Ezio, 481.

LEVÍ-PROVENÇAL, E., 412, 529.

Leyenda de Alejandto, Texto árabe de. 307.

Leyes, Jacobo de las, 250.

Libro de Alexandre and Roman d'Ale-, xandte, 461, 462, 463.

Libro de Apolonio, 216.

Libto de Buen Amor, 79, 528, 557.

Literatura arábigo-española, 318.

Literatura española, Antologia de la. 414.

Litetatuta española, Histotia de la, 188.

Littératute portugaise, La, 430.

Livto de Alportel, O, 334.

Libe d'Ensenyaments delectables, 378.

Loa in Spanish drama, 337.

LONCHAY, Henry, 215, 293, 369.

LONG, Wesley Robertson, 451.

Lope de Vega Carpio, Félix, 100, 141, $154,222,227,260,314,322$,

$323,343,348,358,447,456$,

$472,479,481,538,567$.

LÓPEZ MARTÍNEZ, C., 190.

LÓPEZ DE VEGA, Antonio, 482.

LOURO, Estanco, 344.

LUCA, Giuseppe de, 405, 407.

Lucanot, El conde, 331.

LUCAS-DUBRETON, J., 202.

Lull, Ramón, 391, 324.

LUQUIENS, Frederic Bliss, 530.

LURIA, Max, A., 395.

Luzán, La poética de, 311.

LYNCH, Charles H., 531.

LYNN, Caro, 511.

LLORENS, E. L., 370. 
Mc.

MCCLELLAND, I, L., 483. MACKENSIE, Donald, 168 .

M

MACÉ, Alcide, 9.

Madrid et l'Escorial, 427.

MAGNIN, E., 94.

MAPES, Erwin K., 279, 547.

MARAÑ́N, Gregorio, 335, 431 .

MARASSO, A., 462.

MARDEN, C. Carroll, 216, 297.

Matiana, Juan de, 12, 21, 22, 23, 24 , $30,37,112,137,421,443$.

MARÍN Y OCETE, Antonio, 536.

Marineo Siculo, Lucio, 511.

Maroc, Histoite du, 76, 153, 156.

Mattial in Spain, 331.

Martín Fierro, El, 266.

MARTINENCHE, E., 32, 38, 43, 55 , $73,192$.

MARTINEZ DE BURGOS, M., 152.

MARTÍNEZ DE LA ROSA, 432.

MARTÍNEZ TORNER, E., 203.

MARTÍNEZ Y MARTÍNEZ, Fco., 484.

MARVAUD, A., 172.

MASSAGUER, Enrique, 472.

MATULKA, Bárbara, 298, 327, 371.

MAURA, G., 58.

Maurophitie littéraite en Espagne, La, $409,504,513,544,551,563$, $564,565$.

MAYER, Ernesto, 280.

Meam-Loez, 575.

Medinaceli, Biblioteca del Duque de, 129.

Mejicanismos en el inglés de norteamérica, 247.

MELE, Eugenio, 391.

Melo, Francisco de, 18.

Melo, Francisco Manuel de, 119.

Memorial de diversas hazañas, 41.

Mena y Medtano, Pedro de, 97.

MENÉNDEZ PIDAL, Ramón, 10, 116 , $204,217,224,226,244,299$, $300,321,346,443,453,532$, 568.

Menéndez y Pelayo, Marcelino, 147. 472.

Menosprecio de corte, 152.

MEREDITH, J. A., 347.

MÉRIMÉE, Ernest, $32,38,43,46,55$, $73,191$.

MÉrimée, Henri, 51, 218.

MÉRIMÉE, Paul, 485.
MERRIMAN, R. B., 455.

MESNARD, Pierre, 486.

Mester de Clerecía, Sut le, 554, 579.

Mexican historical novel, 533.

Mexique, La conquête spitituelle du, 438.

MEZ, A., 487.

Michaelis de Vasconcellos, D. Carolina, 307.

MIGUÉlez, P., 140, 261.

Milagros' de Nuestta Señota, 206.

MILLARES CARLO, Agustín, 313.

MILLÉ Y GIMÉNEZ, J., 384, 434.

MIR, Miguel, 95.

Mira de Amescua, 255.

Mistici di Spagna, 407.

Modern Language Review, 294.

MOFFATT, L. G., 426.

MOLDENHAUER, G., 349.

MOLHO, Michael, 575.

Molina, Matia de, 466.

Molina, Titso de, 508, 521.

Molinet, Chroniques de Jean, 525.

MONMARCHÉ, Marcel, 156, 220.

Montalvo y Rodó, 539.

MONTESINOS, José F., 222, 262, 322 , $323,456,567$.

Moot in Spanish literature, The, 275.

MOREL-FATIO, A., 43, 80.

MORÍNIGO, Marcos A., 435.

MORLEY, S. Griswold, 70, 173.

Mosé Arragel de Guadalfajara, Rabi, 234.

MOUNIER, André, 128.

Mozárabes de Toledo, Los, 342.

Mystiques des Pays-Bas, Les, 296.

N

Nebrija, Gtamática castellana de, 253. NORTHUP, G. Tyler, 263, 350.

Nouvelle Revue Internationale, 17.

Novela histórica en España, La, 540.

Novelas ejemplates de Cetvantes, 159. 428.

NYKL, A. R., 372, 413.

\section{$\mathrm{O}$}

Ocantos, Carlos María..., 448 .

Ocampo, Florián de, 84.

Ohio State University, Bulletin, 213.

ONÍs, Federico de, 148.

Ortología castellana de nombres.... 161.

ORUETA, R. de, 97, 157. 
$\mathrm{p}$

Padilla, Lorenzo de, 85.

Pagès, A., 366, 457, 488, 569.

Palacio confuso, El, 538.

Palacios Rubios, Dr., 292.

Palencia, Alfonso de, 41.

PAREJA CASAÑAS, F. M., 458.

Paris et Casa Velásquez, Pierte, 505.

PARRA-PÉREZ, C., 450.

PASTOR, J. F., 351.

PAZ, Julián, $117,129,373,392$, 436.

PEDRELL, F, 44, 245.

Pedro de Artanga, San, 264.

Pere II, el Gran, 175 a.

PEREIRA NOVAES, M., 118.

PEERS, E. Allison, 219, 281, 324.

Pérez, Antonio, 211.

PÉREZ DE AYALA, R., 130.

PÉREZ DE URBEL, Fr. Justo, 447.

PÉREZ PASTOR, Cristóbal, 14.

Peribánez y el Comendador de Ocaña, $154,577$.

PETROF, D. K., 372.

Philippe II ... sa vie et son caractère. 78.

PICÓN-FEBRES, G., 52.

Pierre IV d'Aragón, 569.

PIETSCH, Karl, 246.

Pinheiro da Veiga, Thomé, 68.

plo, Príncipe, 352.

PIRES DE LIMA, A. C., 158, 193.

Poesía juglaresca y juglates, 217.

Poésie épique du siècle d'or, 576.

Polo de Medina, Salvador Jacinto, 382.

PONS, J. S., 353.

Portugal, Le, 15.

PRESTAGE, E., 98, 119, 489.

PRIMICERIO, Elena, 325.

Prince déguisé, Le, 327.

PULIDO Y FERNÁNDEZ, Angel, 33.

\section{Q}

Quijote, Don, 25, 348, 510.

\section{$\mathrm{R}$}

RAMOS Y LOSCERTALES, J, Ma., 301. READ, J. Lloyd, 533.

Reality in Spanish poetty, 548.

Renacimiento del Islam, El, 487.

Renaissance en Catalogne, La, 229.

RENNERT, Hugo A., 141.

REPARAZ, Gonzalo de, 354, 355, 393 , 490.
REPARAz, hijo, Gonzalo de, 374.

RESTREPO, P. Félix, 534.

Revista de Archivos, Bibliotecas y $\mathrm{Mu}$ seos, 62.

Revista Lusitana, 158.

Revue des Universités du Midi, 2, 3.

Revue Hispanique, 141, 20 (nota 1).

Revue Historique de Botdeaux, 42, 175 a, 223 a.

REY, Ct. García, 326.

Rey en su imaginación, El, 222.

REYES, Alfonso, 154.

Reyes Católicos, Los, 290; Crónica de los, 282 ; Documentos correspondientes a los, 170.

REYNIER, G., 131.

RIBELLES COMf́N, José, 394.

RIBERA, Julián, 34, 302.

RICARD, Pr., 156, 220.

RICARD, Robert, 438.

ROBERTS, Graves Baxter, 491.

ROBLES DÉGANO, F., 99.

Rodtigo en la litetatura, El tey, 244.

RODRÍGUEZ MARfN, Francisco, 159.

ROMÁN, Francisco de B. San, 492.

Romancero general, 348.

Romances et chroniques, Rapport entre, 269.

Romances viejos, Flor nueva de, 300 .

Romanische Forschungen, 30.

Romantic movement in Spain, 493.

ROMERA NAVARRo, Miguel, 414.

Ronsatd et les Espagnols, 553.

ROSENBERG, S. L. Millard, 100.

Rousseau in Spanish world, 537.

ROZA DE AMPUDIA, A. de Llano, 155. 459.

Ruiz, Juan, 528.

RUIZ MORCUENDE, Federico, 207.

\section{S}

ST. AMOUR, Sister Mary Paulina, 535.

St. Jean de la Croix, 149, 176.

SAAVEDRA MOLINA, Julio, 547.

SACRAMENTADO, P. Crisógono de Jesús, 375 .

Saint Btaulio of Satagossa, 531.

SAINZ Y RODRfGUEZ, Pedro, $316,335$.

SALADO ALVAREZ, V., 247.

SALARRULLANA DE DIOS, José, 45.

SALINAS, Pedro, 548.

SALVA, V., 101.

San Lorenzo el Real, 416.

SANABRE, Joseph, 383.

Sánchez, Francisco ... el Brocense, 232. 
SÁNCHEZ, Galo, 160, 248.

SÁNCHEZ ALBORNOZ, Cl., 194.

SANCHEZ ALONSO, B., 195, 303, 570.

SÁNCHEZ CANTÓN, F.' J., 174.

Sancho IV de Castilla, 291.

Santa Teresa, 95, 132, 134, 150, 199. 214,312 .

Santillana, Marqués de, 420; Bibliothèque $d u, 35$.

Santo Domingo, Cultura y letras en, 477.

SARGENT, Cecilia Vennard, 356.

SAROÏHANDY, J., 362.

SARRAILH, Jean, 432.

SAVJ LOPEZ, P., 93.

SCHIFF, Mario, 35.

SCUDÉRY, Georges de, 327.

Sefardi, La raza, 33.

Sefer Xaaxuim, 388.

Segovia, Provincia de, 36.

Sephardim, Publications sur les, 515.

Sepúlveda, Juan Ginés de, 233.

"Set" et "Estar". 22, 308.

SERÍs, Homero, II.

SERNA, Juan de la, 188.

SERRA-VILARO, J., 493.

SERRANO, D. Luciano, 264, 328, 357 , 494.

Servet, Miguel... y el Renacimiento, 337.

SHAFFER, Jack, W., 221.

SHOEMAKER, W. H., 495.

Sierra Nevada, Die, 501.

SILVESTRE, Gregorio, 536.

Simancas, Archivo general de, 117.

SOLALINDE, Antonio G., 175, 206.

Soldevia, Ferran, 175 a.

Solemnitats de Barcelona, 383.

SOLENNI, G. de, 358.

Solitudes of Luis Góngora, 377.

SORRENTO, L., 102.

Spain, A brief history of, 402.

Spanish American Literature at Yale University, 530.

Spanish drama before Lope de Vega, 209.

Spanish Empire, The rise of the, 455.

Spanish Mysticism, 219.

SPELL, Jefferson Rea, 537.

STEVENS, Ch. H., 538.

SUNYOL, Dom Gregori Ma., 265.

SWITZEK, Rebecca, 304.

\section{$\mathrm{T}$}

Talavera, Archiprêtre de, 204, 225.

Teatro del mundo, El gran, 542.
Teatro en Valladolid, El, 208.

TERLINGEN, Johannes Hermanus, 571.

Tharaud et Cervantes, Les frères, 142.

Théâtre espagnol, 218.

Thiers, Fondation, 23.

Thity, R., 1.

THOMAS, Lucien-Paul, 376.

THORNDIKE, Lynn, 460.

Ticknor Libraty, 314.

TISCORNIA, Eleuterio F., 266.

Todd Memorial Volumes, 308.

TORO Y GISBERT, Miguel de, 59, 103, 161,415 .

TORRE, Martín de la, 496.

TORRES, M., 359.

Torroella (Pere), Catalan writer, 365.

Tragedia Josephina, 404.

Tragedia por los celos, La, 273.

Tristán de Leonis, El cuento de, 350.

\section{U}

UGARTE, Manuel, 60.

UnAMUNo, Miguel de, 19.

Université de Bordeaux, Etudes hispaniques à $l^{\prime}, 4$; Et le Portugal, 47.

Universidad de Chile, Anales de la, 56.

Universidad de Valladolid, 249.

UREÑA, R. de, 250.

Ustariz, Getónimo de, 128.

\section{V}

Valenciana, Bibliografia de la lengua, 394.

Valera, Diego de, 41, 282.

Valera y Menéndez Pelayo, Epistolario de.... 335.

Valladolid, Universidad de, 249.

VALLEJO, E. Varón, 454.

Vega, Monastetio de, 328.

Vélez de Guevara, Luis, 222.

Venezolana, Literatura, 52.

VERDAGUeR, Mosén Jacinto, 11.

VERGARA Y MARTÍN, G. M. 36.

Vicente de Oviedo, San, 357.

$V i d a$ es sueño, La, 108.

VILA, Salvador, 487.

Villancico, $A$ study of the, 545 .

Vitués, Ctistóbal de, 356.

VISCHER, Elisabeth, 212.

Vitetbe, Annius de, 419.

Viuda de Padilla, 432. 
Vivès . . Exposition. . . 566.

VIVES, D. José, 329.

VOIGT, P., 501 .

$Y$

YO TEN CATE, 572.

Ystorias de Orient, Flor de, 451.

W

$z$

WAGNER, Ch. Ph., 360.

WAGNER, Max Leopold, 395.

Watrigand, H., 2.

WIENER, Leo, 196.

WILSON, Edward Meryon, 377.

WILLIAMS, E. Bucher, 267.

WILLIS, H., 461.

WILLIS, R. S., 462.

WITHERS, A. M., 251.

ZALDUMBIDE, Gonzalo, 539.

Zamora, Gil de, 81.

Zanfoña et Zampoña, 546.

Zapata, Luis, 478.

ZELLERS, Guillermo, 540.

ZARCO CUEVAS, F. J., 416.

Zorrilla, José, 259.

ZUGASTI, J. A., 132.

Zúniga y Avila, Don Luis de, 409.

Zurita, Jerónimo, 502. 
